\title{
The Role of the Protonation State on the Structure and Dynamics of Albumin
}

\author{
Natalia Díaz and Dimas Suárez
}

\section{Supporting Information}

Table S1. $p K a$ values computed for all the ionizable amino acids in the free form of HSA considering different crystal structures (PDB codes: 1AO6, 3JRY, 4G03, and 4EMX) and four dielectric constants for the interior of the protein $\left(\varepsilon_{i n t}\right)$. In bold, we highlight those $p K_{a}$ values that point to a nonstandard protonation state at $\mathrm{pH} 7$ and also those results that suggest deprotonation of the corresponding residue when the $p H$ rises to 9 .

\begin{tabular}{|c|c|c|c|c|c|c|c|c|c|c|c|c|c|c|c|c|}
\hline \multirow{2}{*}{ Residue } & \multicolumn{4}{|c|}{$1 \mathrm{AO6}$ (mol. A, $2.50 \AA$ ) } & \multicolumn{4}{|c|}{ 3JRY (mol. A, $2.30 \AA$ ) } & \multicolumn{4}{|c|}{ 4G03 (mol. A, $2.22 \AA)$} & \multicolumn{4}{|c|}{ 4EMX (mol. A, $2.30 \AA$ ) } \\
\hline & $\varepsilon_{i n t}=4$ & $\varepsilon_{i n t}=6$ & $\varepsilon_{i n t}=10$ & $\varepsilon_{i n t}=20$ & $\varepsilon_{\text {int }}=4$ & $\varepsilon_{i n t}=6$ & $\varepsilon_{i n t}=10$ & $\varepsilon_{i n t}=20$ & $\varepsilon_{i n t}=4$ & $\varepsilon_{i n t}=6$ & $\varepsilon_{\text {int }}=10$ & $\varepsilon_{\text {int }}=20$ & $\varepsilon_{i n t}=4$ & $\varepsilon_{i n t}=6$ & $\varepsilon_{i n t}=10$ & $\varepsilon_{i n t}=20$ \\
\hline His3 & -- & -- & $-{ }_{--}$ & -- & -- & -- & -- & -- & -- & -- & -- & -- & 4.7 & 4.8 & 5.3 & 5.9 \\
\hline Lys4 & -- & -- & - & -- & - & - & - & -- & -- & -- & - & -- & $>12.0$ & $>12.0$ & $>12.0$ & $>12.0$ \\
\hline Glu6 & $<0.0$ & $<0.0$ & 0.1 & 1.0 & $<0.0$ & 0.7 & 0.4 & 1.2 & $<0.0$ & $<0.0$ & 0.4 & 1.1 & $<0.0$ & $<0.0$ & $<0.0$ & $<0.0$ \\
\hline His9 & 4.3 & 6.3 & 8.0 & 8.4 & 9.6 & 9.6 & 9.5 & 9.2 & 3.7 & 5.8 & 7.3 & 7.7 & 10.3 & 9.6 & 9.0 & 8.4 \\
\hline Arg10 & $>12.0$ & $>12.0$ & $>12.0$ & $>12.0$ & $>12.0$ & $>12.0$ & $>12.0$ & $>12.0$ & $>12.0$ & $>12.0$ & $>12.0$ & $>12.0$ & $>12.0$ & $>12.0$ & $>12.0$ & $>12.0$ \\
\hline Lys12 & $>12.0$ & $>12.0$ & $>12.0$ & $>12.0$ & 10.9 & 11.1 & 11.3 & 11.5 & 11.5 & 11.7 & $>12.0$ & $>12.0$ & 11.4 & 11.7 & $>12.0$ & $>12.0$ \\
\hline Asp13 & 9.1 & 6.9 & 5.1 & 4.3 & 7.0 & 5.9 & 4.9 & 4.0 & 7.8 & 6.1 & 4.5 & 3.8 & 6.0 & 4.7 & 3.3 & 2.6 \\
\hline Glu16 & 2.7 & 2.4 & 2.3 & 2.2 & 2.7 & 2.5 & 2.3 & 2.2 & 0.9 & 1.3 & 1.6 & 1.8 & 1.4 & 1.6 & 1.7 & 1.8 \\
\hline Glu17 & 2.8 & 3.0 & 3.1 & 3.2 & 1.7 & 1.9 & 2.1 & 2.3 & 2.1 & 2.3 & 2.5 & 2.6 & 1.7 & 1.7 & 1.8 & 2.1 \\
\hline Lys20 & $>12.0$ & $>12.0$ & $>12.0$ & $>12.0$ & $>12.0$ & $>12.0$ & $>12.0$ & $>12.0$ & 9.5 & 9.9 & 10.3 & 10.6 & 11.6 & 11.7 & $>12.0$ & $>12.0$ \\
\hline Tyr30 & $>12.0$ & $>12.0$ & $>12.0$ & $>12.0$ & $>12.0$ & $>12.0$ & $>12.0$ & $>12.0$ & $>12.0$ & $>12.0$ & $>12.0$ & $>12.0$ & $>12.0$ & $>12.0$ & $>12.0$ & $>12.0$ \\
\hline Cys34 & $>12.0$ & $>12.0$ & $>12.0$ & 10.8 & $>12.0$ & $>12.0$ & 10.9 & 9.7 & $>12.0$ & $>12.0$ & $>12.0$ & 10.5 & $>12.0$ & $>12.0$ & $>12.0$ & 11.4 \\
\hline Glu37 & 3.4 & 3.4 & 3.4 & 3.2 & 3.2 & 3.4 & 3.4 & 3.3 & 3.6 & 3.6 & 3.4 & 3.3 & 3.1 & 3.2 & 3.2 & 3.0 \\
\hline Asp38 & 4.7 & 4.2 & 3.8 & 2.6 & 4.3 & 3.9 & 3.4 & 2.4 & 3.8 & 3.6 & 2.8 & 2.3 & 3.5 & 3.3 & 2.8 & 1.9 \\
\hline His39 & $<0.0$ & $<0.0$ & $<0.0$ & 5.0 & $<0.0$ & $<0.0$ & $<0.0$ & 4.9 & $<0.0$ & $<0.0$ & 1.7 & 5.5 & $<0.0$ & $<0.0$ & $<0.0$ & 5.2 \\
\hline Lys41 & $>12.0$ & $>12.0$ & $>12.0$ & $>12.0$ & $>12.0$ & $>12.0$ & $>12.0$ & $>12.0$ & $>12.0$ & $>12.0$ & $>12.0$ & $>12.0$ & 11.5 & 11.6 & 11.7 & $>12.0$ \\
\hline
\end{tabular}




\begin{tabular}{|c|c|c|c|c|c|c|c|c|c|c|c|c|c|c|c|c|}
\hline Glu45 & 0.5 & 1.1 & 1.5 & 1.8 & 1.8 & 2.1 & 2.3 & 2.3 & 4.3 & 3.8 & 3.3 & 2.9 & 2.8 & 3.0 & 2.9 & 2.7 \\
\hline Glu48 & 3.8 & 3.8 & 3.8 & 3.8 & 4.1 & 4.0 & 3.9 & 3.8 & 3.5 & 3.6 & 3.7 & 3.8 & 3.7 & 3.7 & 3.8 & 3.8 \\
\hline Lys51 & 10.8 & 11.0 & 11.2 & 11.4 & 10.9 & 11.1 & 11.3 & 11.5 & $>12.0$ & $>12.0$ & $>12.0$ & $>12.0$ & $>12.0$ & $>12.0$ & $>12.0$ & $>12.0$ \\
\hline Asp56 & 3.4 & 3.5 & 3.6 & 3.6 & 5.1 & 4.7 & 4.4 & 4.1 & 4.6 & 4.4 & 4.2 & 3.9 & 3.8 & 3.9 & 3.8 & 3.7 \\
\hline Glu57 & 5.9 & 4.9 & 4.2 & 3.7 & 5.4 & 5.2 & 4.3 & 3.6 & 3.5 & 3.3 & 3.2 & 3.2 & 3.6 & 3.0 & 2.8 & 2.7 \\
\hline Glu60 & 3.5 & 3.7 & 3.9 & 4.0 & 5.5 & 5.2 & 4.9 & 4.5 & 4.1 & 4.2 & 4.3 & 4.3 & 7.3 & 6.3 & 5.4 & 4.3 \\
\hline Asp63 & 3.6 & 3.6 & 3.6 & 3.5 & 4.3 & 4.2 & 3.8 & 3.5 & 3.3 & 3.3 & 3.4 & 3.4 & 1.8 & 1.9 & 1.9 & 1.8 \\
\hline Lys64 & $>12.0$ & $>12.0$ & $>12.0$ & $>12.0$ & 10.8 & 11.0 & 11.3 & 11.5 & 10.8 & 11.1 & 11.4 & 11.6 & $>12.0$ & $>12.0$ & $>12.0$ & $>12.0$ \\
\hline His67 & 11.1 & 9.5 & 9.0 & 8.3 & 8.1 & 8.1 & 8.0 & 7.8 & 10.8 & 9.4 & 9.0 & 8.5 & $>12.0$ & 11.0 & 10.1 & 9.2 \\
\hline Asp72 & 0.9 & 1.2 & 1.5 & 1.7 & 1.3 & 1.7 & 1.9 & 1.9 & 1.0 & 1.4 & 1.6 & 1.7 & $<0.0$ & $<0.0$ & $<0.0$ & $<0.0$ \\
\hline Lys73 & $>12.0$ & $>12.0$ & $>12.0$ & $>12.0$ & $>12.0$ & $>12.0$ & $>12.0$ & $>12.0$ & 11.2 & 11.5 & $>12.0$ & $>12.0$ & $>12.0$ & $>12.0$ & $>12.0$ & $>12.0$ \\
\hline Arg81 & $>12.0$ & $>12.0$ & $>12.0$ & $>12.0$ & $>12.0$ & $>12.0$ & $>12.0$ & $>12.0$ & $>12.0$ & $>12.0$ & $>12.0$ & $>12.0$ & $>12.0$ & $>12.0$ & $>12.0$ & $>12.0$ \\
\hline Glu82 & 3.6 & 3.7 & 3.9 & 3.9 & 3.6 & 3.7 & 3.8 & 3.9 & 3.6 & 3.8 & 3.8 & 3.9 & 4.1 & 4.1 & 4.2 & 4.2 \\
\hline Tyr84 & $>12.0$ & $>12.0$ & $>12.0$ & $>12.0$ & $>12.0$ & $>12.0$ & $>12.0$ & $>12.0$ & $>12.0$ & $>12.0$ & $>12.0$ & $>12.0$ & $>12.0$ & $>12.0$ & $>12.0$ & $>12.0$ \\
\hline Glu86 & 3.7 & 3.7 & 3.6 & 3.5 & 4.2 & 4.1 & 4.1 & 4.1 & 3.5 & 3.6 & 3.6 & 3.6 & 3.6 & 3.6 & 3.6 & 3.5 \\
\hline Asp89 & 2.6 & 2.8 & 2.9 & 3.0 & 1.8 & 1.9 & 2.1 & 2.2 & 2.6 & 2.8 & 2.9 & 3.0 & 0.8 & 0.9 & 1.0 & 1.1 \\
\hline Lys93 & 9.7 & 10.3 & 10.8 & 11.3 & 9.3 & 9.9 & 10.5 & 11.0 & 11.1 & 11.2 & 11.4 & 11.6 & $>12.0$ & $>12.0$ & $>12.0$ & $>12.0$ \\
\hline Glu95 & $<0.0$ & $<0.0$ & $<0.0$ & 0.1 & $<0.0$ & $<0.0$ & $<0.0$ & 0.5 & $<0.0$ & $<0.0$ & $<0.0$ & 0.5 & $<0.0$ & $<0.0$ & $<0.0$ & $<0.0$ \\
\hline Glu97 & 4.9 & 4.7 & 4.5 & 4.3 & 5.3 & 5.0 & 4.7 & 4.4 & 3.5 & 3.7 & 3.8 & 3.9 & 7.0 & 5.8 & 4.7 & 3.5 \\
\hline Arg98 & $>12.0$ & $>12.0$ & $>12.0$ & $>12.0$ & $>12.0$ & $>12.0$ & $>12.0$ & $>12.0$ & $>12.0$ & $>12.0$ & $>12.0$ & $>12.0$ & $>12.0$ & $>12.0$ & $>12.0$ & $>12.0$ \\
\hline Glu100 & 3.1 & 3.1 & 3.1 & 3.1 & 3.8 & 3.6 & 3.5 & 3.5 & 5.1 & 4.2 & 3.6 & 3.3 & 1.6 & 1.7 & 1.7 & 1.9 \\
\hline His105 & 7.6 & 7.5 & 7.4 & 7.3 & 3.6 & 5.2 & 6.1 & 6.7 & 7.4 & 7.4 & 7.4 & 7.4 & 8.6 & 8.5 & 8.4 & 8.3 \\
\hline Lys106 & $>12.0$ & $>12.0$ & $>12.0$ & $>12.0$ & $>12.0$ & $>12.0$ & $>12.0$ & $>12.0$ & $>12.0$ & $>12.0$ & $>12.0$ & $>12.0$ & $>12.0$ & $>12.0$ & $>12.0$ & $>12.0$ \\
\hline Asp107 & 0.2 & 0.4 & 0.5 & 0.6 & 1.0 & 0.9 & 0.8 & 0.7 & $<0.0$ & 0.2 & 0.3 & 0.5 & 0.1 & 0.3 & 0.3 & 0.3 \\
\hline Asp108 & $<0.0$ & $<0.0$ & $<0.0$ & $<0.0$ & $<0.0$ & $<0.0$ & $<0.0$ & $<0.0$ & $<0.0$ & $<0.0$ & $<0.0$ & $<0.0$ & $<0.0$ & $<0.0$ & $<0.0$ & $<0.0$ \\
\hline Arg114 & $>12.0$ & $>12.0$ & $>12.0$ & $>12.0$ & $>12.0$ & $>12.0$ & $>12.0$ & $>12.0$ & $>12.0$ & $>12.0$ & $>12.0$ & $>12.0$ & $>12.0$ & $>12.0$ & $>12.0$ & $>12.0$ \\
\hline Arg117 & $>12.0$ & $>12.0$ & $>12.0$ & $>12.0$ & $>12.0$ & $>12.0$ & $>12.0$ & $>12.0$ & $>12.0$ & $>12.0$ & $>12.0$ & $>12.0$ & $>12.0$ & $>12.0$ & $>12.0$ & $>12.0$ \\
\hline Glu119 & 4.6 & 4.5 & 4.3 & 4.0 & 3.5 & 3.6 & 3.7 & 3.8 & 5.0 & 4.8 & 4.5 & 4.3 & 3.7 & 3.7 & 3.8 & 3.8 \\
\hline Asp121 & 1.3 & 1.6 & 1.9 & 2.0 & 1.1 & 1.6 & 2.0 & 2.3 & 1.8 & 2.0 & 2.1 & 2.3 & 3.1 & 3.0 & 2.9 & 2.8 \\
\hline His128 & 7.6 & 7.7 & 7.8 & 7.7 & 6.1 & 6.7 & 7.0 & 7.3 & 6.6 & 6.8 & 7.0 & 7.0 & 6.5 & 6.7 & 6.9 & 7.0 \\
\hline Asp129 & 3.1 & 3.2 & 3.3 & 3.4 & 3.4 & 3.5 & 3.5 & 3.5 & 3.2 & 3.3 & 3.3 & 3.4 & 3.2 & 3.3 & 3.4 & 3.4 \\
\hline Glu131 & 5.9 & 5.3 & 4.7 & 4.1 & 3.9 & 3.6 & 3.5 & 3.3 & 5.4 & 4.8 & 4.2 & 3.7 & 4.8 & 4.3 & 3.9 & 3.5 \\
\hline
\end{tabular}




\begin{tabular}{|c|c|c|c|c|c|c|c|c|c|c|c|c|c|c|c|c|}
\hline Glu132 & 1.9 & 2.0 & 2.1 & 2.3 & 3.9 & 3.8 & 3.7 & 3.6 & 2.8 & 1.8 & 1.2 & 1.1 & 2.2 & 2.2 & 2.3 & 2.3 \\
\hline Lys136 & 9.0 & 9.7 & 10.3 & 11.1 & 7.8 & 8.6 & 9.4 & 10.4 & 5.5 & 7.5 & 8.8 & 10.3 & 7.9 & 8.8 & 9.7 & 10.9 \\
\hline Lys137 & 10.6 & 10.7 & 10.9 & 11.4 & 10.0 & 10.3 & 10.7 & 11.3 & 10.8 & 11.0 & 11.2 & 11.7 & 10.5 & 10.7 & 10.9 & 11.5 \\
\hline Tyr138 & $>12.0$ & $>12.0$ & $>12.0$ & $>12.0$ & $>12.0$ & $>12.0$ & $>12.0$ & $>12.0$ & $>12.0$ & $>12.0$ & $>12.0$ & $>12.0$ & $>12.0$ & $>12.0$ & $>12.0$ & $>12.0$ \\
\hline Tyr140 & $>12.0$ & $>12.0$ & $>12.0$ & $>12.0$ & $>12.0$ & $>12.0$ & $>12.0$ & $>12.0$ & $>12.0$ & $>12.0$ & $>12.0$ & $>12.0$ & $>12.0$ & $>12.0$ & $>12.0$ & $>12.0$ \\
\hline Glu141 & $<0.0$ & $<0.0$ & $<0.0$ & $<0.0$ & $<0.0$ & $<0.0$ & $<0.0$ & $<0.0$ & $<0.0$ & $<0.0$ & $<0.0$ & $<0.0$ & $<0.0$ & $<0.0$ & $<0.0$ & $<0.0$ \\
\hline Arg144 & $>12.0$ & $>12.0$ & $>12.0$ & $>12.0$ & $>12.0$ & $>12.0$ & $>12.0$ & $>12.0$ & $>12.0$ & $>12.0$ & $>12.0$ & $>12.0$ & $>12.0$ & $>12.0$ & $>12.0$ & $>12.0$ \\
\hline Arg145 & $>12.0$ & $>12.0$ & $>12.0$ & $>12.0$ & $>12.0$ & $>12.0$ & $>12.0$ & $>12.0$ & $>12.0$ & $>12.0$ & $>12.0$ & $>12.0$ & $>12.0$ & $>12.0$ & $>12.0$ & $>12.0$ \\
\hline His146 & $<0.0$ & $<0.0$ & $<0.0$ & 2.7 & $<0.0$ & $<0.0$ & 0.6 & 2.9 & $<0.0$ & $<0.0$ & $<0.0$ & 2.1 & $<0.0$ & $<0.0$ & 1.2 & 3.2 \\
\hline Tyr148 & $>12.0$ & $>12.0$ & $>12.0$ & $>12.0$ & $>12.0$ & $>12.0$ & $>12.0$ & $>12.0$ & $>12.0$ & $>12.0$ & $>12.0$ & $>12.0$ & $>12.0$ & $>12.0$ & $>12.0$ & $>12.0$ \\
\hline Tyr150 & $>12.0$ & $>12.0$ & $>12.0$ & $>12.0$ & $>12.0$ & $>12.0$ & $>12.0$ & $>12.0$ & $>12.0$ & $>12.0$ & $>12.0$ & $>12.0$ & $>12.0$ & $>12.0$ & $>12.0$ & $>12.0$ \\
\hline Glu153 & $<0.0$ & $<0.0$ & $<0.0$ & $<0.0$ & $<0.0$ & $<0.0$ & $<0.0$ & $<0.0$ & $<0.0$ & $<0.0$ & $<0.0$ & $<0.0$ & $<0.0$ & $<0.0$ & $<0.0$ & $<0.0$ \\
\hline Lys159 & $>12.0$ & $>12.0$ & $>12.0$ & $>12.0$ & 9.5 & 9.9 & 10.3 & 10.7 & 10.3 & 10.8 & 11.3 & $>12.0$ & 11.5 & 11.4 & 11.4 & 11.5 \\
\hline $\operatorname{Arg} 160$ & $>12.0$ & $>12.0$ & $>12.0$ & $>12.0$ & $>12.0$ & $>12.0$ & $>12.0$ & $>12.0$ & $>12.0$ & $>12.0$ & $>12.0$ & $>12.0$ & $>12.0$ & $>12.0$ & $>12.0$ & $>12.0$ \\
\hline Tyr161 & $>12.0$ & $>12.0$ & $>12.0$ & $>12.0$ & $>12.0$ & $>12.0$ & 11.6 & 10.3 & $>12.0$ & $>12.0$ & $>12.0$ & 11.5 & $>12.0$ & $>12.0$ & $>12.0$ & 10.9 \\
\hline Lys162 & $>12.0$ & $>12.0$ & $>12.0$ & $>12.0$ & $>12.0$ & $>12.0$ & $>12.0$ & $>12.0$ & $>12.0$ & $>12.0$ & $>12.0$ & $>12.0$ & 11.4 & 11.7 & $>12.0$ & $>12.0$ \\
\hline Glu167 & $<0.0$ & $<0.0$ & $<0.0$ & 0.2 & 3.1 & 3.2 & 3.3 & 3.4 & $<0.0$ & $<0.0$ & $<0.0$ & $<0.0$ & 2.1 & 2.5 & 2.8 & 3.0 \\
\hline Asp173 & 0.5 & 0.2 & 0.1 & 0.1 & 3.0 & 3.1 & 3.2 & 3.2 & $<0.0$ & $<0.0$ & $<0.0$ & $<0.0$ & 3.2 & 3.2 & 3.2 & 3.2 \\
\hline Lys174 & $>12.0$ & $>12.0$ & $>12.0$ & $>12.0$ & 11.1 & 11.3 & 11.4 & 11.6 & 10.6 & 11.1 & 11.6 & $>12.0$ & 11.3 & 11.3 & 11.4 & 11.5 \\
\hline Lys181 & 9.8 & 11.0 & $>12.0$ & $>12.0$ & $>12.0$ & $>12.0$ & $>12.0$ & $>12.0$ & $>12.0$ & $>12.0$ & $>12.0$ & $>12.0$ & $>12.0$ & $>12.0$ & $>12.0$ & $>12.0$ \\
\hline Asp183 & $<0.0$ & $<0.0$ & $<0.0$ & $<0.0$ & $<0.0$ & 0.3 & 0.8 & 1.1 & 0.3 & $<0.0$ & $<0.0$ & $<0.0$ & 0.3 & 0.4 & 0.6 & 0.8 \\
\hline Glu184 & 2.2 & 2.2 & 2.1 & 2.0 & 4.2 & 3.9 & 3.7 & 3.5 & 3.5 & 3.1 & 2.8 & 2.6 & 4.3 & 3.9 & 3.6 & 3.3 \\
\hline Arg186 & $>12.0$ & $>12.0$ & $>12.0$ & $>12.0$ & $>12.0$ & $>12.0$ & $>12.0$ & $>12.0$ & $>12.0$ & $>12.0$ & $>12.0$ & $>12.0$ & $>12.0$ & $>12.0$ & $>12.0$ & $>12.0$ \\
\hline Asp187 & $<0.0$ & $<0.0$ & $<0.0$ & $<0.0$ & 1.1 & 0.3 & $<0.0$ & $<0.0$ & $<0.0$ & $<0.0$ & $<0.0$ & $<0.0$ & $<0.0$ & $<0.0$ & $<0.0$ & $<0.0$ \\
\hline Glu188 & $<0.0$ & $<0.0$ & $<0.0$ & $<0.0$ & $<0.0$ & $<0.0$ & $<0.0$ & $<0.0$ & $<0.0$ & $<0.0$ & $<0.0$ & $<0.0$ & $<0.0$ & $<0.0$ & $<0.0$ & $<0.0$ \\
\hline Lys190 & 5.5 & 6.5 & 7.6 & 8.8 & 5.8 & 6.7 & 7.6 & 8.7 & 4.7 & 6.0 & 7.3 & 8.9 & 8.1 & 8.5 & 8.8 & 10.0 \\
\hline Lys195 & 8.4 & 10.6 & $>12.0$ & $>12.0$ & 11.1 & 11.3 & 11.8 & $>12.0$ & 9.9 & 11.5 & $>12.0$ & $>12.0$ & 5.8 & 7.1 & 8.6 & 9.5 \\
\hline Arg197 & $>12.0$ & $>12.0$ & $>12.0$ & $>12.0$ & $>12.0$ & $>12.0$ & $>12.0$ & $>12.0$ & $>12.0$ & $>12.0$ & $>12.0$ & $>12.0$ & $>12.0$ & $>12.0$ & $>12.0$ & $>12.0$ \\
\hline Lys199 & $<0.0$ & 1.6 & 6.1 & 9.0 & $<0.0$ & 4.0 & 6.9 & 9.3 & $<0.0$ & 1.8 & 6.1 & 9.1 & $<0.0$ & $<0.0$ & 5.9 & 9.7 \\
\hline Lys205 & 11.5 & 11.5 & 11.6 & $>12.0$ & $>12.0$ & $>12.0$ & $>12.0$ & $>12.0$ & $>12.0$ & $>12.0$ & $>12.0$ & $>12.0$ & $>12.0$ & $>12.0$ & $>12.0$ & $>12.0$ \\
\hline Glu208 & 2.6 & 2.6 & 2.5 & 2.5 & 2.6 & 2.6 & 2.6 & 2.7 & 2.8 & 2.7 & 2.7 & 2.7 & 1.5 & 1.6 & 1.6 & 1.7 \\
\hline Arg209 & $>12.0$ & $>12.0$ & $>12.0$ & $>12.0$ & $>12.0$ & $>12.0$ & $>12.0$ & $>12.0$ & $>12.0$ & $>12.0$ & $>12.0$ & $>12.0$ & $>12.0$ & $>12.0$ & $>12.0$ & $>12.0$ \\
\hline
\end{tabular}




\begin{tabular}{|c|c|c|c|c|c|c|c|c|c|c|c|c|c|c|c|c|}
\hline Lys212 & 10.7 & 10.9 & 11.2 & 11.4 & 10.6 & 10.8 & 11.1 & 11.3 & 10.1 & 10.5 & 10.9 & 11.3 & 10.7 & 10.9 & 11.3 & 11.7 \\
\hline Arg218 & $>12.0$ & $>12.0$ & $>12.0$ & $>12.0$ & $>12.0$ & $>12.0$ & $>12.0$ & $>12.0$ & $>12.0$ & $>12.0$ & $>12.0$ & $>12.0$ & $>12.0$ & $>12.0$ & $>12.0$ & $>12.0$ \\
\hline Arg222 & $>12.0$ & $>12.0$ & $>12.0$ & $>12.0$ & $>12.0$ & $>12.0$ & $>12.0$ & $>12.0$ & $>12.0$ & $>12.0$ & $>12.0$ & $>12.0$ & $>12.0$ & $>12.0$ & $>12.0$ & $>12.0$ \\
\hline Lys225 & $>12.0$ & $>12.0$ & $>12.0$ & $>12.0$ & 11.4 & 11.5 & 11.7 & 11.8 & $>12.0$ & $>12.0$ & $>12.0$ & $>12.0$ & $>12.0$ & $>12.0$ & $>12.0$ & $>12.0$ \\
\hline Glu227 & 4.1 & 4.2 & 4.2 & 4.1 & 3.6 & 3.7 & 3.9 & 4.1 & 3.9 & 4.1 & 4.2 & 4.2 & 3.8 & 4.0 & 4.1 & 4.2 \\
\hline Glu230 & 2.9 & 2.9 & 2.9 & 3.1 & 6.8 & 5.8 & 4.9 & 4.1 & 3.9 & 3.6 & 3.4 & 3.4 & 4.2 & 3.8 & 3.5 & 3.4 \\
\hline Lys233 & $>12.0$ & $>12.0$ & $>12.0$ & $>12.0$ & $>12.0$ & $>12.0$ & $>12.0$ & $>12.0$ & $>12.0$ & $>12.0$ & $>12.0$ & $>12.0$ & $>12.0$ & $>12.0$ & $>12.0$ & $>12.0$ \\
\hline Asp237 & 2.0 & 1.5 & 1.2 & 1.4 & 0.8 & 0.7 & 1.0 & 1.3 & 0.0 & 0.1 & 0.4 & 0.9 & $<0.0$ & 0.0 & 0.2 & 0.6 \\
\hline Lys240 & $>12.0$ & $>12.0$ & $>12.0$ & $>12.0$ & $>12.0$ & $>12.0$ & $>12.0$ & $>12.0$ & $>12.0$ & $>12.0$ & $>12.0$ & $>12.0$ & $>12.0$ & $>12.0$ & $>12.0$ & $>12.0$ \\
\hline His242 & $<0.0$ & $<0.0$ & $<0.0$ & $<0.0$ & $<0.0$ & $<0.0$ & $<0.0$ & $<0.0$ & $<0.0$ & $<0.0$ & $<0.0$ & $<0.0$ & $<0.0$ & $<0.0$ & $<0.0$ & $<0.0$ \\
\hline Glu244 & $>12.0$ & $>12.0$ & 8.4 & 5.5 & $>12.0$ & $>12.0$ & 8.6 & 5.5 & $>12.0$ & $>12.0$ & 9.6 & 6.2 & $>12.0$ & $>12.0$ & 9.7 & 5.6 \\
\hline His247 & 5.8 & 6.1 & 6.5 & 7.0 & 6.7 & 6.9 & 6.9 & 7.2 & 3.2 & 4.8 & 5.6 & 6.3 & 6.7 & 6.9 & 7.0 & 7.4 \\
\hline Asp249 & $<0.0$ & $<0.0$ & $<0.0$ & $<0.0$ & $<0.0$ & $<0.0$ & $<0.0$ & $<0.0$ & $<0.0$ & $<0.0$ & $<0.0$ & $<0.0$ & $<0.0$ & $<0.0$ & $<0.0$ & $<0.0$ \\
\hline Glu252 & 1.5 & 1.6 & 1.7 & 1.9 & 2.3 & 2.0 & 1.8 & 2.0 & 1.9 & 1.9 & 2.0 & 2.3 & $<0.0$ & $<0.0$ & $<0.0$ & $<0.0$ \\
\hline Asp255 & $<0.0$ & $<0.0$ & $<0.0$ & 0.5 & $<0.0$ & $<0.0$ & 0.1 & 1.0 & $<0.0$ & $<0.0$ & $<0.0$ & 0.1 & $<0.0$ & $<0.0$ & $<0.0$ & $<0.0$ \\
\hline Asp256 & 7.3 & 5.9 & 4.4 & 3.2 & 8.2 & 6.3 & 4.6 & 2.9 & 8.0 & 6.1 & 3.6 & 1.8 & 8.3 & 6.0 & 3.1 & 1.4 \\
\hline $\operatorname{Arg} 257$ & $>12.0$ & $>12.0$ & $>12.0$ & $>12.0$ & $>12.0$ & $>12.0$ & $>12.0$ & $>12.0$ & $>12.0$ & $>12.0$ & $>12.0$ & $>12.0$ & $>12.0$ & $>12.0$ & $>12.0$ & $>12.0$ \\
\hline Asp259 & 2.9 & 3.0 & 3.0 & 3.1 & 4.0 & 3.7 & 3.7 & 3.6 & 4.3 & 3.8 & 3.8 & 3.6 & 3.5 & 3.2 & 3.1 & 3.1 \\
\hline Lys262 & $>12.0$ & $>12.0$ & $>12.0$ & $>12.0$ & $>12.0$ & $>12.0$ & $>12.0$ & $>12.0$ & $>12.0$ & $>12.0$ & $>12.0$ & $>12.0$ & $>12.0$ & $>12.0$ & $>12.0$ & $>12.0$ \\
\hline Tyr263 & $>12.0$ & $>12.0$ & $>12.0$ & $>12.0$ & $>12.0$ & $>12.0$ & $>12.0$ & $>12.0$ & $>12.0$ & $>12.0$ & $>12.0$ & $>12.0$ & $>12.0$ & $>12.0$ & $>12.0$ & $>12.0$ \\
\hline Glu266 & 5.3 & 4.9 & 4.5 & 4.2 & 4.8 & 4.4 & 4.0 & 3.8 & 4.7 & 4.4 & 4.2 & 4.0 & 5.7 & 5.1 & 4.7 & 4.3 \\
\hline Asp269 & 3.8 & 3.9 & 3.9 & 3.9 & 3.1 & 3.1 & 3.1 & 3.2 & 3.5 & 3.6 & 3.7 & 3.7 & 3.5 & 3.6 & 3.7 & 3.7 \\
\hline Lys274 & 11.6 & $>12.0$ & $>12.0$ & $>12.0$ & $>12.0$ & $>12.0$ & $>12.0$ & $>12.0$ & $>12.0$ & $>12.0$ & $>12.0$ & $>12.0$ & $>12.0$ & $>12.0$ & $>12.0$ & $>12.0$ \\
\hline Lys276 & $>12.0$ & $>12.0$ & $>12.0$ & $>12.0$ & $>12.0$ & $>12.0$ & 11.7 & 11.6 & $>12.0$ & $>12.0$ & $>12.0$ & $>12.0$ & 11.2 & 11.2 & 11.2 & 11.2 \\
\hline Glu277 & 1.8 & 2.1 & 2.3 & 2.6 & 5.8 & 5.1 & 4.4 & 3.9 & 2.4 & 2.4 & 2.4 & 2.4 & 2.7 & 2.9 & 3.1 & 3.3 \\
\hline Glu280 & 4.5 & 4.3 & 4.1 & 3.9 & 4.5 & 4.3 & 4.1 & 4.0 & 4.6 & 4.4 & 4.2 & 4.1 & 5.1 & 4.7 & 4.4 & 4.1 \\
\hline Lys281 & $>12.0$ & $>12.0$ & $>12.0$ & $>12.0$ & $>12.0$ & $>12.0$ & $>12.0$ & $>12.0$ & $>12.0$ & $>12.0$ & $>12.0$ & $>12.0$ & 11.0 & 11.5 & 11.7 & $>12.0$ \\
\hline Glu285 & $<0.0$ & $<0.0$ & $<0.0$ & $<0.0$ & $<0.0$ & $<0.0$ & 0.4 & 0.9 & $<0.0$ & $<0.0$ & $<0.0$ & $<0.0$ & $<0.0$ & $<0.0$ & 0.4 & 0.9 \\
\hline Lys286 & 11.2 & 11.5 & 11.7 & $>12.0$ & 9.5 & 10.0 & 10.5 & 11.1 & 10.1 & 10.6 & 11.1 & 11.6 & 9.9 & 10.6 & 11.0 & 11.5 \\
\hline His288 & $>12.0$ & 8.6 & 7.9 & 7.3 & 7.8 & 7.4 & 7.0 & 6.9 & 9.7 & 7.9 & 7.3 & 6.9 & $>12.0$ & 9.4 & 7.9 & 7.3 \\
\hline Glu292 & $<0.0$ & $<0.0$ & $<0.0$ & $<0.0$ & $<0.0$ & $<0.0$ & $<0.0$ & $<0.0$ & $<0.0$ & $<0.0$ & $<0.0$ & $<0.0$ & $<0.0$ & $<0.0$ & $<0.0$ & $<0.0$ \\
\hline Glu294 & 2.1 & 1.9 & 1.7 & 1.9 & 3.6 & 3.4 & 3.2 & 3.1 & 3.0 & 2.6 & 2.3 & 2.3 & 3.9 & 3.6 & 3.0 & 2.8 \\
\hline
\end{tabular}




\begin{tabular}{|c|c|c|c|c|c|c|c|c|c|c|c|c|c|c|c|c|}
\hline Asp296 & $<0.0$ & $<0.0$ & $<0.0$ & $<0.0$ & $<0.0$ & $<0.0$ & 0.5 & 1.1 & $<0.0$ & $<0.0$ & $<0.0$ & $<0.0$ & $<0.0$ & $<0.0$ & $<0.0$ & 0.3 \\
\hline Glu297 & 2.6 & 2.8 & 3.0 & 3.1 & 4.8 & 4.5 & 4.3 & 4.1 & 2.8 & 3.0 & 3.1 & 3.1 & 3.1 & 3.2 & 3.3 & 3.3 \\
\hline Asp301 & 3.7 & 3.8 & 3.8 & 3.8 & 3.8 & 3.8 & 3.8 & 3.8 & 3.7 & 3.7 & 3.7 & 3.7 & 3.8 & 3.8 & 3.8 & 3.8 \\
\hline Asp308 & 4.8 & 4.4 & 4.1 & 3.8 & 3.4 & 3.4 & 3.4 & 3.4 & 4.6 & 4.3 & 4.1 & 3.7 & 3.7 & 3.7 & 3.6 & 3.5 \\
\hline Glu311 & 4.1 & 4.2 & 4.2 & 4.2 & 4.2 & 4.1 & 4.1 & 4.0 & 4.0 & 4.1 & 4.1 & 4.1 & 4.6 & 4.4 & 4.2 & 4.1 \\
\hline Lys313 & 11.0 & 11.0 & 11.0 & 11.0 & 11.1 & 11.1 & 11.0 & 11.0 & 11.0 & 11.0 & 11.0 & 11.0 & 11.2 & 11.1 & 11.1 & 11.1 \\
\hline Asp314 & 2.3 & 2.5 & 2.7 & 2.8 & 2.3 & 2.5 & 2.7 & 2.9 & 3.2 & 3.1 & 3.1 & 3.1 & 2.3 & 2.6 & 2.8 & 2.9 \\
\hline Lys317 & $>12.0$ & 11.7 & 11.6 & 11.5 & 11.3 & 11.3 & 11.4 & 11.4 & 11.8 & 11.7 & 11.7 & 11.7 & 11.5 & 11.6 & 11.7 & 11.7 \\
\hline Tyr319 & $>12.0$ & $>12.0$ & $>12.0$ & $>12.0$ & $>12.0$ & $>12.0$ & $>12.0$ & $>12.0$ & $>12.0$ & $>12.0$ & $>12.0$ & $>12.0$ & $>12.0$ & $>12.0$ & $>12.0$ & $>12.0$ \\
\hline Glu321 & 5.0 & 4.7 & 4.5 & 4.3 & 5.2 & 5.0 & 4.7 & 4.5 & 5.6 & 5.2 & 4.9 & 4.6 & 5.1 & 4.8 & 4.5 & 4.2 \\
\hline Lys323 & 11.7 & 11.6 & 11.6 & 11.6 & $>12.0$ & $>12.0$ & $>12.0$ & $>12.0$ & $>12.0$ & $>12.0$ & 11.8 & 11.7 & $>12.0$ & $>12.0$ & $>12.0$ & $>12.0$ \\
\hline Asp324 & 1.0 & 1.1 & 1.3 & 1.4 & 0.7 & 0.9 & 1.1 & 1.4 & 0.5 & 0.9 & 1.2 & 1.6 & 3.1 & 2.4 & 1.8 & 1.5 \\
\hline Tyr332 & $>12.0$ & $>12.0$ & $>12.0$ & $>12.0$ & $>12.0$ & $>12.0$ & $>12.0$ & $>12.0$ & $>12.0$ & $>12.0$ & $>12.0$ & $>12.0$ & $>12.0$ & $>12.0$ & $>12.0$ & $>12.0$ \\
\hline Glu333 & $<0.0$ & 0.6 & 1.1 & 1.7 & 0.8 & 1.2 & 1.6 & 2.0 & $<0.0$ & 0.6 & 1.2 & 1.9 & $<0.0$ & 0.6 & 1.2 & 1.8 \\
\hline Tyr334 & $>12.0$ & $>12.0$ & $>12.0$ & $>12.0$ & $>12.0$ & $>12.0$ & $>12.0$ & 11.1 & $>12.0$ & $>12.0$ & $>12.0$ & 11.3 & $>12.0$ & $>12.0$ & $>12.0$ & 11.7 \\
\hline Arg336 & $>12.0$ & $>12.0$ & $>12.0$ & $>12.0$ & $>12.0$ & $>12.0$ & $>12.0$ & $>12.0$ & $>12.0$ & $>12.0$ & $>12.0$ & $>12.0$ & $>12.0$ & $>12.0$ & $>12.0$ & $>12.0$ \\
\hline Arg337 & $>12.0$ & $>12.0$ & $>12.0$ & $>12.0$ & $>12.0$ & $>12.0$ & $>12.0$ & $>12.0$ & $>12.0$ & $>12.0$ & $>12.0$ & $>12.0$ & $>12.0$ & $>12.0$ & $>12.0$ & $>12.0$ \\
\hline His338 & 7.8 & 7.6 & 7.5 & 7.4 & 7.5 & 7.4 & 7.3 & 7.3 & 7.3 & 7.3 & 7.2 & 7.1 & 7.1 & 7.1 & 7.0 & 6.9 \\
\hline Asp340 & $<0.0$ & 0.3 & 0.6 & 1.0 & 0.9 & 1.0 & 1.1 & 1.3 & 1.2 & 1.3 & 1.4 & 1.4 & 1.0 & 1.1 & 1.1 & 1.3 \\
\hline Tyr341 & $>12.0$ & $>12.0$ & $>12.0$ & $>12.0$ & $>12.0$ & $>12.0$ & $>12.0$ & $>12.0$ & $>12.0$ & $>12.0$ & $>12.0$ & $>12.0$ & $>12.0$ & $>12.0$ & $>12.0$ & $>12.0$ \\
\hline Arg348 & $>12.0$ & $>12.0$ & $>12.0$ & $>12.0$ & $>12.0$ & $>12.0$ & $>12.0$ & $>12.0$ & $>12.0$ & $>12.0$ & $>12.0$ & $>12.0$ & $>12.0$ & $>12.0$ & $>12.0$ & $>12.0$ \\
\hline Lys351 & 10.7 & 10.8 & 10.9 & 11.1 & 8.4 & 9.0 & 9.5 & 10.2 & 10.6 & 10.6 & 10.8 & 10.9 & 11.2 & 11.3 & 11.5 & 11.7 \\
\hline Tyr353 & $>12.0$ & $>12.0$ & $>12.0$ & $>12.0$ & $>12.0$ & $>12.0$ & $>12.0$ & $>12.0$ & $>12.0$ & $>12.0$ & $>12.0$ & $>12.0$ & $>12.0$ & $>12.0$ & $>12.0$ & $>12.0$ \\
\hline Glu354 & 1.0 & 0.9 & 1.1 & 1.4 & 3.5 & 3.1 & 2.7 & 2.5 & 3.1 & 2.4 & 2.1 & 2.0 & 2.6 & 2.8 & 2.6 & 2.5 \\
\hline Glu358 & 3.2 & 3.4 & 3.5 & 3.6 & 1.9 & 2.4 & 2.9 & 3.1 & 2.8 & 3.2 & 3.4 & 3.5 & $<0.0$ & 0.2 & 1.2 & 2.1 \\
\hline Lys359 & 10.7 & 10.8 & 10.8 & 10.9 & 11.1 & 11.1 & 11.1 & 11.2 & 10.9 & 11.0 & 11.2 & 11.3 & 11.3 & 11.4 & 11.4 & 11.5 \\
\hline Asp365 & 2.9 & 3.0 & 3.2 & 3.2 & 2.9 & 3.0 & 3.0 & 3.0 & 3.0 & 3.0 & 3.1 & 3.2 & 3.2 & 3.1 & 3.0 & 3.0 \\
\hline His367 & 5.3 & 5.9 & 6.4 & 6.7 & 8.7 & 8.3 & 7.9 & 7.6 & 8.1 & 8.0 & 7.9 & 7.9 & 7.5 & 7.4 & 7.4 & 7.3 \\
\hline Glu368 & 3.7 & 3.8 & 3.9 & 4.0 & 4.0 & 3.9 & 3.9 & 3.9 & 3.8 & 4.0 & 4.1 & 4.2 & 3.5 & 3.7 & 3.8 & 3.9 \\
\hline Tyr370 & $>12.0$ & $>12.0$ & $>12.0$ & $>12.0$ & $>12.0$ & $>12.0$ & $>12.0$ & $>12.0$ & $>12.0$ & $>12.0$ & $>12.0$ & $>12.0$ & $>12.0$ & $>12.0$ & $>12.0$ & $>12.0$ \\
\hline Lys372 & 11.1 & 11.1 & 11.2 & 11.3 & 11.7 & 11.8 & $>12.0$ & $>12.0$ & 11.1 & 11.2 & 11.2 & 11.3 & 11.1 & 11.1 & 11.2 & 11.3 \\
\hline Asp375 & 3.1 & 3.1 & 3.1 & 3.1 & 2.5 & 2.6 & 2.7 & 2.8 & 3.6 & 3.4 & 3.3 & 3.2 & 3.2 & 3.1 & 3.1 & 3.0 \\
\hline
\end{tabular}




\begin{tabular}{|c|c|c|c|c|c|c|c|c|c|c|c|c|c|c|c|c|}
\hline Glu376 & 4.8 & 4.5 & 4.3 & 4.1 & 6.5 & 5.8 & 5.1 & 4.6 & 5.9 & 5.2 & 4.6 & 4.0 & 5.5 & 5.1 & 4.7 & 4.3 \\
\hline Lys378 & 11.0 & 11.1 & 11.2 & 11.4 & 11.2 & 11.3 & 11.6 & $>12.0$ & 10.7 & 10.9 & 11.0 & 11.2 & 10.8 & 10.9 & 11.0 & 11.1 \\
\hline Glu382 & 4.6 & 4.4 & 4.1 & 3.9 & 3.9 & 3.7 & 3.5 & 3.4 & 4.4 & 4.2 & 4.0 & 3.8 & 4.6 & 4.4 & 4.2 & 4.0 \\
\hline Glu383 & $<0.0$ & $<0.0$ & 0.5 & 0.9 & $<0.0$ & $<0.0$ & $<0.0$ & $<0.0$ & $<0.0$ & 0.2 & 0.7 & 1.1 & $<0.0$ & $<0.0$ & 0.3 & 0.9 \\
\hline Lys389 & 10.5 & 10.6 & 10.8 & 11.0 & 11.7 & 11.8 & $>12.0$ & $>12.0$ & 10.4 & 10.7 & 11.0 & 11.3 & 10.5 & 10.9 & 11.1 & 11.4 \\
\hline Glu393 & 3.0 & 3.0 & 3.0 & 3.0 & 3.1 & 2.9 & 2.7 & 2.6 & 3.6 & 3.4 & 3.4 & 3.3 & 3.6 & 3.5 & 3.3 & 3.2 \\
\hline Glu396 & 2.9 & 2.7 & 2.3 & 2.2 & 4.7 & 4.4 & 4.0 & 3.7 & 3.7 & 3.1 & 2.4 & 1.9 & 5.6 & 4.9 & 4.3 & 3.7 \\
\hline Glu400 & $<0.0$ & $<0.0$ & $<0.0$ & $<0.0$ & 1.4 & 1.3 & 1.2 & 1.1 & $<0.0$ & $<0.0$ & $<0.0$ & $<0.0$ & $<0.0$ & $<0.0$ & $<0.0$ & 0.2 \\
\hline Tyr401 & $>12.0$ & $>12.0$ & $>12.0$ & 10.2 & $>12.0$ & $>12.0$ & $>12.0$ & 8.6 & $>12.0$ & $>12.0$ & $>12.0$ & 8.4 & $>12.0$ & $>12.0$ & $>12.0$ & 9.1 \\
\hline Lys402 & $>12.0$ & $>12.0$ & $>12.0$ & $>12.0$ & 10.7 & 10.8 & 10.9 & 11.1 & $>12.0$ & $>12.0$ & $>12.0$ & $>12.0$ & 10.7 & 10.9 & 11.0 & 11.2 \\
\hline Arg410 & 9.7 & 11.2 & $>12.0$ & $>12.0$ & $>12.0$ & $>12.0$ & $>12.0$ & $>12.0$ & 9.0 & 10.4 & $>12.0$ & $>12.0$ & $>12.0$ & $>12.0$ & $>12.0$ & $>12.0$ \\
\hline Tyr411 & $>12.0$ & $>12.0$ & $>12.0$ & 8.6 & $>12.0$ & $>12.0$ & 8.9 & 8.4 & $>12.0$ & $>12.0$ & 11.6 & 8.2 & $>12.0$ & $>12.0$ & 9.8 & 8.8 \\
\hline Lys413 & 11.0 & 10.7 & 10.5 & 10.8 & $>12.0$ & $>12.0$ & $>12.0$ & $>12.0$ & 8.8 & 9.3 & 9.9 & 10.6 & 9.2 & 9.8 & 10.2 & 10.8 \\
\hline Lys414 & 6.8 & 7.9 & 9.9 & 11.7 & 7.0 & 8.6 & $>12.0$ & $>12.0$ & 1.9 & 5.4 & 7.3 & 10.1 & 9.1 & 9.5 & 11.7 & $>12.0$ \\
\hline Glu425 & $<0.0$ & $<0.0$ & $<0.0$ & $<0.0$ & $<0.0$ & $<0.0$ & $<0.0$ & $<0.0$ & $<0.0$ & $<0.0$ & $<0.0$ & $<0.0$ & $<0.0$ & $<0.0$ & $<0.0$ & $<0.0$ \\
\hline $\operatorname{Arg} 428$ & $>12.0$ & $>12.0$ & $>12.0$ & $>12.0$ & $>12.0$ & $>12.0$ & $>12.0$ & $>12.0$ & $>12.0$ & $>12.0$ & $>12.0$ & $>12.0$ & $>12.0$ & $>12.0$ & $>12.0$ & $>12.0$ \\
\hline Lys432 & 8.1 & 8.7 & 9.4 & 10.8 & 9.3 & 9.5 & 9.8 & 10.8 & 7.5 & 8.1 & 9.0 & 10.8 & 6.7 & 7.9 & 9.2 & 11.1 \\
\hline Lys436 & 11.2 & 11.4 & 11.5 & $>12.0$ & 8.1 & 9.0 & 9.9 & $>12.0$ & 11.6 & 11.8 & $>12.0$ & $>12.0$ & 9.7 & 10.5 & 11.0 & $>12.0$ \\
\hline Lys439 & 11.4 & 11.5 & 11.5 & 11.6 & 11.1 & 11.1 & 11.2 & 11.2 & 9.5 & 10.1 & 10.6 & 11.1 & 11.0 & 11.1 & 11.1 & 11.1 \\
\hline His440 & $<0.0$ & 1.0 & 2.8 & 4.3 & 0.9 & 2.1 & 3.4 & 4.6 & $<0.0$ & 0.6 & 2.7 & 4.4 & 0.4 & 1.8 & 3.1 & 4.5 \\
\hline Glu442 & 3.1 & 3.1 & 3.1 & 3.1 & 2.9 & 3.0 & 3.0 & 3.1 & 2.5 & 2.6 & 2.5 & 2.6 & 2.9 & 2.9 & 2.9 & 3.0 \\
\hline Lys444 & 9.4 & 9.9 & 10.2 & 10.6 & 9.4 & 9.8 & 10.2 & 10.6 & 9.6 & 10.0 & 10.3 & 10.7 & 9.2 & 9.6 & 10.1 & 10.4 \\
\hline Arg445 & $>12.0$ & $>12.0$ & $>12.0$ & $>12.0$ & $>12.0$ & $>12.0$ & $>12.0$ & $>12.0$ & $>12.0$ & $>12.0$ & $>12.0$ & $>12.0$ & $>12.0$ & $>12.0$ & $>12.0$ & $>12.0$ \\
\hline Glu450 & $<0.0$ & $<0.0$ & $<0.0$ & $<0.0$ & $<0.0$ & $<0.0$ & $<0.0$ & $<0.0$ & $<0.0$ & $<0.0$ & $<0.0$ & $<0.0$ & $<0.0$ & $<0.0$ & $<0.0$ & $<0.0$ \\
\hline Asp451 & $<0.0$ & $<0.0$ & $<0.0$ & $<0.0$ & $<0.0$ & $<0.0$ & $<0.0$ & $<0.0$ & $<0.0$ & $<0.0$ & $<0.0$ & $<0.0$ & $<0.0$ & $<0.0$ & $<0.0$ & $<0.0$ \\
\hline Tyr452 & $>12.0$ & $>12.0$ & $>12.0$ & $>12.0$ & $>12.0$ & $>12.0$ & $>12.0$ & $>12.0$ & $>12.0$ & $>12.0$ & $>12.0$ & $>12.0$ & $>12.0$ & $>12.0$ & $>12.0$ & $\begin{array}{l}10.7 \\
\end{array}$ \\
\hline His464 & $<0.0$ & 2.3 & 4.7 & 5.8 & $<0.0$ & $<0.0$ & 2.0 & 4.8 & $<0.0$ & $<0.0$ & 0.8 & 4.4 & $<0.0$ & $<0.0$ & 0.4 & 4.4 \\
\hline Glu465 & 3.7 & 2.7 & 1.9 & 1.9 & 1.8 & 1.8 & 1.3 & 1.3 & 1.8 & 1.7 & 1.4 & 1.2 & 2.3 & 2.0 & 1.3 & 0.7 \\
\hline Lys466 & $>12.0$ & $>12.0$ & $>12.0$ & $>12.0$ & 11.1 & 11.4 & 11.7 & $>12.0$ & $>12.0$ & $>12.0$ & $>12.0$ & $>12.0$ & $>12.0$ & $>12.0$ & $>12.0$ & $>12.0$ \\
\hline Asp471 & 2.2 & 2.3 & 2.4 & 2.5 & 2.3 & 2.5 & 2.5 & 2.3 & 2.3 & 2.5 & 2.6 & 2.4 & 2.2 & 2.5 & 2.6 & 2.5 \\
\hline $\operatorname{Arg} 472$ & $>12.0$ & $>12.0$ & $>12.0$ & $>12.0$ & $>12.0$ & $>12.0$ & $>12.0$ & $>12.0$ & $>12.0$ & $>12.0$ & $>12.0$ & $>12.0$ & $>12.0$ & $>12.0$ & $>12.0$ & $>12.0$ \\
\hline Lys475 & 10.0 & 10.2 & 10.4 & 10.7 & $>12.0$ & $>12.0$ & $>12.0$ & $>12.0$ & 10.4 & 10.5 & 10.7 & 10.9 & 11.5 & 11.5 & 11.5 & 11.6 \\
\hline
\end{tabular}




\begin{tabular}{|c|c|c|c|c|c|c|c|c|c|c|c|c|c|c|c|c|}
\hline Glu479 & 3.6 & 3.5 & 3.4 & 3.3 & 1.8 & 2.0 & 2.1 & 2.2 & 3.9 & 3.7 & 3.5 & 3.3 & 2.5 & 2.5 & 2.5 & 2.5 \\
\hline Arg484 & $>12.0$ & $>12.0$ & $>12.0$ & $>12.0$ & $>12.0$ & $>12.0$ & $>12.0$ & $>12.0$ & $>12.0$ & $>12.0$ & $>12.0$ & $>12.0$ & $>12.0$ & $>12.0$ & $>12.0$ & $>12.0$ \\
\hline $\operatorname{Arg} 485$ & $>12.0$ & $>12.0$ & $>12.0$ & $>12.0$ & $>12.0$ & $>12.0$ & $>12.0$ & $>12.0$ & $>12.0$ & $>12.0$ & $>12.0$ & $>12.0$ & $>12.0$ & $>12.0$ & $>12.0$ & $>12.0$ \\
\hline Glu492 & 2.2 & 2.3 & 2.4 & 2.5 & $<0.0$ & $<0.0$ & $<0.0$ & $<0.0$ & 3.7 & 3.3 & 3.3 & 3.4 & 3.1 & 3.1 & 3.2 & 3.3 \\
\hline Asp494 & $<0.0$ & $<0.0$ & 0.6 & 1.1 & 0.0 & 0.2 & 0.0 & 0.2 & 0.4 & 0.7 & 0.9 & 1.2 & 0.2 & 0.7 & 1.1 & 1.3 \\
\hline Glu495 & 4.2 & 4.1 & 4.0 & 3.9 & 2.6 & 2.0 & 1.8 & 1.7 & 5.2 & 4.6 & 4.2 & 3.9 & 4.7 & 4.5 & 4.3 & 4.1 \\
\hline Tyr497 & $>12.0$ & $>12.0$ & $>12.0$ & $>12.0$ & $>12.0$ & $>12.0$ & $>12.0$ & $>12.0$ & $>12.0$ & $>12.0$ & $>12.0$ & 11.5 & $>12.0$ & $>12.0$ & $>12.0$ & 11.3 \\
\hline Lys500 & 11.1 & 11.0 & 10.9 & 10.9 & $>12.0$ & $>12.0$ & $>12.0$ & $>12.0$ & 10.7 & 10.7 & 10.8 & 10.9 & $>12.0$ & $>12.0$ & $>12.0$ & $>12.0$ \\
\hline Glu501 & 3.9 & 3.9 & 3.9 & 3.9 & 3.2 & 3.3 & 3.4 & 3.4 & 4.3 & 3.9 & 3.8 & 3.8 & 3.7 & 3.7 & 3.8 & 3.8 \\
\hline Glu505 & 3.5 & 3.5 & 3.6 & 3.6 & 2.6 & 2.9 & 3.2 & 3.3 & 3.1 & 3.2 & 3.3 & 3.4 & 3.6 & 3.7 & 3.7 & 3.8 \\
\hline His510 & 7.3 & 7.4 & 7.5 & 7.5 & $<0.0$ & 1.3 & 4.7 & 5.7 & 6.8 & 6.9 & 6.9 & 7.0 & 7.9 & 7.5 & 7.3 & 7.1 \\
\hline Asp512 & 1.9 & 1.9 & 2.0 & 2.0 & 5.1 & 4.5 & 2.6 & 2.5 & 2.5 & 2.3 & 2.1 & 2.0 & 1.4 & 1.4 & 1.5 & 1.6 \\
\hline Glu518 & 0.9 & 0.2 & $<0.0$ & $<0.0$ & 2.5 & 2.4 & 2.2 & 2.1 & $<0.0$ & $<0.0$ & $<0.0$ & $<0.0$ & 2.8 & 1.8 & 1.5 & 1.4 \\
\hline Lys519 & $>12.0$ & $>12.0$ & $>12.0$ & $>12.0$ & 11.0 & 10.8 & 10.8 & 10.9 & 8.1 & 8.4 & 8.8 & 9.4 & 0.7 & 4.6 & 6.6 & 8.5 \\
\hline Glu520 & $<0.0$ & $<0.0$ & $<0.0$ & $<0.0$ & 0.3 & 0.8 & 1.2 & 1.5 & $<0.0$ & $<0.0$ & $<0.0$ & $<0.0$ & $<0.0$ & $<0.0$ & 0.0 & 0.5 \\
\hline Arg521 & $>12.0$ & $>12.0$ & $>12.0$ & $>12.0$ & $>12.0$ & $>12.0$ & $>12.0$ & $>12.0$ & $>12.0$ & $>12.0$ & $>12.0$ & $>12.0$ & $>12.0$ & $>12.0$ & $>12.0$ & $>12.0$ \\
\hline Lys524 & 10.1 & 10.3 & 10.6 & 10.9 & 8.3 & 9.0 & 9.7 & 10.4 & 10.5 & 10.7 & 10.9 & 11.1 & 9.4 & 9.9 & 10.3 & 10.7 \\
\hline Lys525 & 7.9 & 9.3 & 11.1 & $>12.0$ & 7.2 & 8.1 & 9.6 & 11.7 & 6.1 & 8.0 & 10.8 & $>12.0$ & 7.0 & 8.1 & 9.4 & $>12.0$ \\
\hline Glu531 & 0.7 & 0.8 & 1.1 & 1.4 & $<0.0$ & $<0.0$ & 0.2 & 0.6 & $<0.0$ & $<0.0$ & $<0.0$ & 0.2 & $<0.0$ & $<0.0$ & $<0.0$ & 0.6 \\
\hline Lys534 & $>12.0$ & $>12.0$ & $>12.0$ & $>12.0$ & $>12.0$ & $>12.0$ & $>12.0$ & $>12.0$ & $>12.0$ & $>12.0$ & $>12.0$ & $>12.0$ & $>12.0$ & $>12.0$ & $>12.0$ & $>12.0$ \\
\hline His535 & 7.5 & 7.2 & 7.2 & 7.2 & 4.7 & 5.4 & 5.8 & 6.1 & 3.1 & 4.6 & 5.5 & 6.1 & 4.3 & 5.2 & 5.9 & 6.3 \\
\hline Lys536 & 8.7 & 10.2 & 11.3 & $>12.0$ & $>12.0$ & $>12.0$ & $>12.0$ & $>12.0$ & $>12.0$ & $>12.0$ & $>12.0$ & $>12.0$ & 11.1 & 11.2 & 11.2 & 11.2 \\
\hline Lys538 & 10.9 & 10.9 & 10.9 & 10.9 & 8.4 & 9.1 & 9.8 & 10.7 & 10.5 & 10.8 & 10.9 & 11.2 & 10.7 & 10.7 & 10.6 & 10.7 \\
\hline Lys541 & 10.2 & 10.3 & 10.3 & 10.4 & 7.8 & 8.1 & 8.7 & 9.4 & 10.5 & 10.5 & 10.5 & 10.5 & 7.6 & 8.2 & 8.8 & 9.4 \\
\hline Glu542 & 2.5 & 2.8 & 3.0 & 3.1 & 1.4 & 1.6 & 1.7 & 1.9 & 2.2 & 2.4 & 2.7 & 2.9 & 2.8 & 2.7 & 2.7 & 2.7 \\
\hline Lys545 & 9.2 & 9.5 & 9.9 & 10.6 & 11.5 & 11.5 & 11.6 & 11.7 & 7.7 & 8.6 & 9.5 & 10.3 & 9.7 & 10.3 & 10.7 & 11.1 \\
\hline Asp549 & 2.9 & 2.2 & 1.9 & 1.8 & 2.6 & 2.3 & 2.1 & 2.0 & 2.9 & 2.1 & 1.5 & 1.2 & 1.5 & 1.6 & 1.8 & 2.0 \\
\hline Asp550 & 5.9 & 5.0 & 4.3 & 3.5 & 5.4 & 4.9 & 4.5 & 4.1 & 7.0 & 5.5 & 4.4 & 3.5 & $>12.0$ & 9.2 & 6.8 & 5.1 \\
\hline Glu556 & 3.0 & 2.7 & 2.4 & 2.3 & 2.8 & 2.8 & 2.8 & 2.9 & 2.5 & 2.3 & 2.0 & 1.8 & 3.1 & 3.1 & 3.1 & 3.1 \\
\hline Lys557 & 11.8 & $>12.0$ & $>12.0$ & $>12.0$ & 11.3 & 11.3 & 11.3 & 11.4 & 11.3 & 11.6 & 11.8 & $>12.0$ & $>12.0$ & $>12.0$ & $>12.0$ & $>12.0$ \\
\hline Lys560 & $>12.0$ & $>12.0$ & $>12.0$ & $>12.0$ & 10.5 & 10.6 & 10.7 & 10.8 & $>12.0$ & $>12.0$ & $>12.0$ & $>12.0$ & 11.6 & 11.5 & 11.3 & 11.2 \\
\hline Asp562 & 3.7 & 3.6 & 3.6 & 3.5 & 4.0 & 3.9 & 3.9 & 3.8 & 4.3 & 4.1 & 3.8 & 3.6 & 4.0 & 4.0 & 4.0 & 3.9 \\
\hline
\end{tabular}




\begin{tabular}{|c|c|c|c|c|c|c|c|c|c|c|c|c|c|c|c|c|}
\hline Asp563 & 1.9 & 2.1 & 2.3 & 2.4 & 2.3 & 2.6 & 2.9 & 3.0 & 0.8 & 1.3 & 1.7 & 2.0 & 0.1 & 1.1 & 1.8 & 2.4 \\
\hline Lys564 & 11.4 & 11.4 & 11.4 & 11.4 & 11.5 & 11.5 & 11.5 & 11.6 & 11.1 & 11.2 & 11.2 & 11.3 & 11.0 & 11.1 & 11.1 & 11.2 \\
\hline Glu565 & 3.6 & 3.8 & 3.8 & 3.9 & 4.2 & 4.3 & 4.2 & 4.1 & 3.4 & 3.6 & 3.7 & 3.9 & 3.8 & 3.9 & 4.0 & 4.0 \\
\hline Glu570 & 3.7 & 3.8 & 3.8 & 3.8 & 3.2 & 3.4 & 3.5 & 3.6 & 3.7 & 3.8 & 3.8 & 3.8 & 4.1 & 4.1 & 4.2 & 4.3 \\
\hline Glu571 & 4.5 & 4.3 & 4.1 & 4.0 & 3.5 & 3.4 & 3.3 & 3.2 & 5.0 & 4.6 & 4.4 & 4.2 & 7.0 & 6.0 & 5.1 & 4.3 \\
\hline Lys573 & 10.8 & 10.9 & 10.9 & 10.9 & 8.0 & 9.0 & 9.8 & 10.4 & 10.9 & 10.9 & 11.0 & 11.0 & 10.5 & 10.6 & 10.7 & 10.7 \\
\hline Lys574 & 10.6 & 10.7 & 10.7 & 10.8 & 10.4 & 10.7 & 10.9 & 11.1 & 10.4 & 10.6 & 10.7 & 10.9 & 10.8 & 11.2 & 11.2 & 11.2 \\
\hline \multirow{2}{*}{ Residue } & \multicolumn{4}{|c|}{$1 \mathrm{AO6}(\mathrm{mol} . \mathrm{B}, 2.50 \AA)$} & \multicolumn{4}{|c|}{ 3JRY (mol. B, $2.30 \AA)$} & \multicolumn{4}{|c|}{ 4G03 (mol. B, $2.22 \AA)$} & \multicolumn{4}{|c|}{ 4EMX (mol. B, $2.30 \AA)$} \\
\hline & $\varepsilon_{\text {int }}=4$ & $\varepsilon_{\text {int }}=6$ & $\varepsilon_{\text {int }}=10$ & $\varepsilon_{\text {int }}=20$ & $\varepsilon_{\text {int }}=4$ & $\varepsilon_{\text {int }}=6$ & $\varepsilon_{\text {int }}=10$ & $\varepsilon_{\text {int }}=20$ & $\varepsilon_{\text {int }}=4$ & Eint $=6$ & $\varepsilon_{\text {int }}=10$ & $\varepsilon_{\text {int }}=20$ & $\varepsilon_{\text {int }}=4$ & $\varepsilon_{\text {int }}=6$ & $\varepsilon_{\text {int }}=10$ & $\varepsilon_{\text {int }}=20$ \\
\hline Lys4 & -- & -- & -- & -- & -- & -- & -- & -- & -- & -- & -- & -- & $>12.0$ & $>12.0$ & $>12.0$ & $>12.0$ \\
\hline Glu6 & $<0.0$ & $<0.0$ & $<0.0$ & 0.5 & $<0.0$ & 0.5 & 0.4 & 1.2 & $<0.0$ & $<0.0$ & 0.1 & 1.0 & $<0.0$ & $<0.0$ & $<0.0$ & $<0.0$ \\
\hline His9 & 9.8 & 9.8 & 9.5 & 9.1 & 8.7 & 9.3 & 9.3 & 9.1 & 3.4 & 5.2 & 7.2 & 7.7 & 4.1 & 5.3 & 7.1 & 7.7 \\
\hline Arg10 & $>12.0$ & $>12.0$ & $>12.0$ & $>12.0$ & $>12.0$ & $>12.0$ & $>12.0$ & $>12.0$ & $>12.0$ & $>12.0$ & $>12.0$ & $>12.0$ & $>12.0$ & $>12.0$ & $>12.0$ & $>12.0$ \\
\hline Lys12 & 11.6 & 11.8 & $>12.0$ & $>12.0$ & 10.9 & 11.1 & 11.3 & 11.4 & $>12.0$ & $>12.0$ & $>12.0$ & $>12.0$ & 11.2 & 11.6 & $>12.0$ & $>12.0$ \\
\hline Asp13 & 7.6 & 6.2 & 5.2 & 4.3 & 6.5 & 5.2 & 4.4 & 3.8 & 8.5 & 7.0 & 5.0 & 4.2 & 9.3 & 7.4 & 5.0 & 4.1 \\
\hline Glu16 & 2.3 & 2.3 & 2.3 & 2.3 & 2.5 & 2.4 & 2.3 & 2.2 & 1.4 & 1.7 & 1.9 & 2.0 & 1.7 & 1.9 & 2.0 & 2.1 \\
\hline Glu17 & 3.2 & 3.3 & 3.3 & 3.3 & 1.7 & 1.9 & 2.1 & 2.3 & 1.5 & 1.5 & 1.6 & 1.8 & 0.9 & 1.1 & 1.4 & 1.7 \\
\hline Lys20 & $>12.0$ & $>12.0$ & $>12.0$ & $>12.0$ & $>12.0$ & $>12.0$ & $>12.0$ & $>12.0$ & 11.4 & 11.6 & 11.8 & $>12.0$ & 10.4 & 10.7 & 11.0 & 11.2 \\
\hline Tyr30 & $>12.0$ & $>12.0$ & $>12.0$ & $>12.0$ & $>12.0$ & $>12.0$ & $>12.0$ & $>12.0$ & $>12.0$ & $>12.0$ & $>12.0$ & $>12.0$ & $>12.0$ & $>12.0$ & $>12.0$ & $>12.0$ \\
\hline Cys34 & $>12.0$ & $>12.0$ & $>12.0$ & 11.1 & $>12.0$ & $>12.0$ & 10.7 & 9.6 & $>12.0$ & $>12.0$ & $>12.0$ & 11.0 & $>12.0$ & $>12.0$ & $>12.0$ & $>12.0$ \\
\hline Glu37 & 4.0 & 3.9 & 3.7 & 3.4 & 3.4 & 3.5 & 3.5 & 3.3 & 3.8 & 3.7 & 3.5 & 3.2 & 4.2 & 4.1 & 3.8 & 3.5 \\
\hline Asp38 & 3.7 & 3.6 & 3.3 & 2.5 & 4.0 & 3.7 & 3.4 & 2.4 & 3.5 & 3.4 & 3.2 & 2.4 & 3.8 & 3.4 & 2.9 & 2.1 \\
\hline His39 & $<0.0$ & $<0.0$ & $<0.0$ & 5.1 & $<0.0$ & $<0.0$ & $<0.0$ & 4.7 & $<0.0$ & $<0.0$ & $<0.0$ & 4.8 & $<0.0$ & $<0.0$ & 0.6 & 5.4 \\
\hline Lys41 & 11.5 & 11.5 & 11.5 & 11.6 & $>12.0$ & $>12.0$ & $>12.0$ & $>12.0$ & $>12.0$ & $>12.0$ & $>12.0$ & $>12.0$ & 11.6 & 11.6 & 11.6 & 11.8 \\
\hline Glu45 & 2.1 & 2.3 & 2.4 & 2.4 & 2.1 & 2.3 & 2.4 & 2.3 & 3.6 & 3.3 & 3.0 & 2.7 & 3.3 & 3.3 & 3.3 & 3.1 \\
\hline Glu48 & 4.2 & 4.1 & 4.0 & 3.9 & 4.1 & 4.0 & 3.9 & 3.8 & 3.6 & 3.7 & 3.8 & 3.8 & 3.9 & 3.9 & 3.9 & 3.8 \\
\hline Lys51 & 10.9 & 11.0 & 11.2 & 11.4 & 11.1 & 11.3 & 11.4 & 11.6 & $>12.0$ & $>12.0$ & $>12.0$ & $>12.0$ & $>12.0$ & $>12.0$ & 11.7 & 11.7 \\
\hline Asp56 & 4.6 & 4.3 & 4.0 & 3.8 & 5.2 & 4.8 & 4.4 & 4.1 & 4.7 & 4.4 & 4.1 & 3.8 & 4.5 & 4.2 & 3.9 & 3.7 \\
\hline Glu57 & 3.1 & 3.2 & 3.4 & 3.5 & 5.4 & 5.1 & 4.3 & 3.6 & 3.4 & 3.2 & 3.2 & 3.2 & 3.1 & 2.9 & 2.9 & 2.9 \\
\hline Glu60 & 3.5 & 3.7 & 3.8 & 3.9 & 5.4 & 5.1 & 4.8 & 4.5 & 4.4 & 4.4 & 4.3 & 4.3 & 4.4 & 4.4 & 4.3 & 4.3 \\
\hline Asp63 & 3.7 & 3.7 & 3.6 & 3.5 & 4.4 & 4.3 & 3.9 & 3.5 & 3.9 & 3.8 & 3.7 & 3.6 & 2.8 & 2.9 & 2.9 & 2.8 \\
\hline Lys64 & 11.3 & 11.4 & 11.6 & 11.7 & 10.8 & 11.0 & 11.3 & 11.5 & 11.2 & 11.4 & 11.5 & 11.7 & $>12.0$ & $>12.0$ & $>12.0$ & $>12.0$ \\
\hline
\end{tabular}




\begin{tabular}{|c|c|c|c|c|c|c|c|c|c|c|c|c|c|c|c|c|}
\hline His67 & $>12.0$ & 10.5 & 9.6 & 8.6 & 7.7 & 7.7 & 7.7 & 7.6 & 9.7 & 9.0 & 8.7 & 8.3 & 9.4 & 9.2 & 9.0 & 8.8 \\
\hline Asp72 & 1.7 & 1.8 & 1.9 & 1.9 & 1.1 & 1.5 & 1.7 & 1.9 & 0.7 & 1.1 & 1.4 & 1.6 & 1.9 & 2.0 & 2.0 & 1.9 \\
\hline Lys73 & $>12.0$ & $>12.0$ & $>12.0$ & $>12.0$ & $>12.0$ & $>12.0$ & $>12.0$ & $>12.0$ & 11.3 & 11.5 & 11.7 & $>12.0$ & $>12.0$ & $>12.0$ & $>12.0$ & $>12.0$ \\
\hline Arg81 & $>12.0$ & $>12.0$ & $>12.0$ & $>12.0$ & $>12.0$ & $>12.0$ & $>12.0$ & $>12.0$ & $>12.0$ & $>12.0$ & $>12.0$ & $>12.0$ & $>12.0$ & $>12.0$ & $>12.0$ & $>12.0$ \\
\hline Glu82 & 3.8 & 3.9 & 4.0 & 4.0 & 3.6 & 3.7 & 3.8 & 3.9 & 3.4 & 3.6 & 3.8 & 3.9 & 4.0 & 4.1 & 4.1 & 4.1 \\
\hline Tyr84 & $>12.0$ & $>12.0$ & $>12.0$ & $>12.0$ & $>12.0$ & $>12.0$ & $>12.0$ & $>12.0$ & $>12.0$ & $>12.0$ & $>12.0$ & $>12.0$ & $>12.0$ & $>12.0$ & $>12.0$ & $>12.0$ \\
\hline Glu86 & 4.0 & 4.0 & 3.8 & 3.7 & 4.2 & 4.1 & 4.1 & 4.0 & 3.2 & 3.4 & 3.5 & 3.5 & 4.9 & 4.7 & 4.6 & 4.4 \\
\hline Asp89 & 2.3 & 2.5 & 2.8 & 2.9 & 1.6 & 1.8 & 2.0 & 2.2 & 1.7 & 2.1 & 2.4 & 2.6 & 1.6 & 1.6 & 1.7 & 1.8 \\
\hline Lys93 & 10.4 & 10.9 & 11.2 & 11.5 & 9.5 & 10.0 & 10.5 & 11.0 & 11.0 & 11.2 & 11.4 & 11.6 & $>12.0$ & $>12.0$ & $>12.0$ & $>12.0$ \\
\hline Glu95 & $<0.0$ & $<0.0$ & $<0.0$ & 0.8 & $<0.0$ & $<0.0$ & $<0.0$ & 0.3 & $<0.0$ & $<0.0$ & $<0.0$ & 0.2 & $<0.0$ & $<0.0$ & $<0.0$ & $<0.0$ \\
\hline Glu97 & 3.1 & 3.3 & 3.5 & 3.6 & 5.2 & 4.9 & 4.6 & 4.4 & 5.4 & 5.1 & 4.8 & 4.5 & 0.0 & 0.0 & $<0.0$ & 0.0 \\
\hline Arg98 & $>12.0$ & $>12.0$ & $>12.0$ & $>12.0$ & $>12.0$ & $>12.0$ & $>12.0$ & $>12.0$ & $>12.0$ & $>12.0$ & $>12.0$ & $>12.0$ & $>12.0$ & $>12.0$ & $>12.0$ & $>12.0$ \\
\hline Glu100 & 1.9 & 2.0 & 2.1 & 2.2 & 3.7 & 3.6 & 3.5 & 3.4 & 4.7 & 4.1 & 3.8 & 3.6 & 1.2 & 0.1 & $<0.0$ & $<0.0$ \\
\hline His105 & 7.4 & 7.4 & 7.5 & 7.4 & 4.3 & 5.4 & 6.2 & 6.7 & 7.6 & 7.5 & 7.5 & 7.4 & 10.7 & 10.1 & 9.7 & 9.3 \\
\hline Lys106 & $>12.0$ & $>12.0$ & $>12.0$ & $>12.0$ & $>12.0$ & $>12.0$ & $>12.0$ & $>12.0$ & $>12.0$ & $>12.0$ & $>12.0$ & $>12.0$ & $>12.0$ & $>12.0$ & $>12.0$ & $>12.0$ \\
\hline Asp107 & $<0.0$ & 0.2 & 0.2 & 0.3 & $<0.0$ & $<0.0$ & $<0.0$ & 0.2 & $<0.0$ & $<0.0$ & $<0.0$ & 0.1 & $<0.0$ & $<0.0$ & $<0.0$ & $<0.0$ \\
\hline \begin{tabular}{|l|} 
Asp108 \\
\end{tabular} & $<0.0$ & $<0.0$ & $<0.0$ & $<0.0$ & $<0.0$ & $<0.0$ & $<0.0$ & $<0.0$ & $<0.0$ & $<0.0$ & $<0.0$ & $<0.0$ & $<0.0$ & $<0.0$ & $<0.0$ & $<0.0$ \\
\hline Arg114 & $>12.0$ & $>12.0$ & $>12.0$ & $>12.0$ & $>12.0$ & $>12.0$ & $>12.0$ & $>12.0$ & $>12.0$ & $>12.0$ & $>12.0$ & $>12.0$ & $>12.0$ & $>12.0$ & $>12.0$ & $>12.0$ \\
\hline Arg117 & $>12.0$ & $>12.0$ & $>12.0$ & $>12.0$ & $>12.0$ & $>12.0$ & $>12.0$ & $>12.0$ & $>12.0$ & $>12.0$ & $>12.0$ & $>12.0$ & $>12.0$ & $>12.0$ & $>12.0$ & $>12.0$ \\
\hline \begin{tabular}{|l|} 
Glu119 \\
\end{tabular} & 6.2 & 5.7 & 5.2 & 4.7 & 3.5 & 3.6 & 3.7 & 3.8 & 4.1 & 4.0 & 4.0 & 3.9 & 3.6 & 3.7 & 3.7 & 3.8 \\
\hline Asp121 & 1.9 & 2.0 & 2.1 & 2.2 & 0.9 & 1.5 & 1.9 & 2.3 & 2.0 & 2.0 & 2.1 & 2.2 & 3.0 & 2.9 & 2.8 & 2.7 \\
\hline His128 & 7.6 & 7.7 & 7.7 & 7.7 & 6.2 & 6.6 & 7.0 & 7.3 & 4.8 & 5.8 & 6.3 & 6.6 & 6.1 & 6.5 & 6.8 & 7.0 \\
\hline \begin{tabular}{|l|} 
Asp129 \\
\end{tabular} & 3.2 & 3.3 & 3.3 & 3.4 & 3.3 & 3.4 & 3.5 & 3.5 & 3.2 & 3.3 & 3.3 & 3.4 & 3.4 & 3.4 & 3.4 & 3.4 \\
\hline Glu131 & 4.3 & 4.0 & 3.7 & 3.4 & 4.5 & 3.9 & 3.6 & 3.4 & 5.2 & 4.3 & 3.7 & 3.2 & 5.5 & 4.8 & 4.4 & 4.0 \\
\hline Glu132 & 0.6 & 0.8 & 1.1 & 1.3 & 3.7 & 3.8 & 3.8 & 3.6 & 1.1 & 1.3 & 1.6 & 1.9 & 3.2 & 3.1 & 3.1 & 3.0 \\
\hline Lys136 & 9.4 & 10.0 & 10.6 & 11.3 & 7.8 & 8.6 & 9.4 & 10.3 & 8.1 & 9.0 & 9.9 & 11.0 & 6.4 & 7.7 & 8.9 & 10.1 \\
\hline \begin{tabular}{|l|} 
Lys 137 \\
\end{tabular} & 10.0 & 10.2 & 10.5 & 11.1 & 9.9 & 10.2 & 10.7 & 11.2 & 10.7 & 10.9 & 11.2 & 11.7 & 10.6 & 10.7 & 10.8 & 11.4 \\
\hline \begin{tabular}{|l|} 
Tyr138 \\
\end{tabular} & $>12.0$ & $>12.0$ & $>12.0$ & $>12.0$ & $>12.0$ & $>12.0$ & $>12.0$ & $>12.0$ & $>12.0$ & $>12.0$ & $>12.0$ & $>12.0$ & $>12.0$ & $>12.0$ & $>12.0$ & $>12.0$ \\
\hline Tyr140 & $>12.0$ & $>12.0$ & $>12.0$ & $>12.0$ & $>12.0$ & $>12.0$ & $>12.0$ & $>12.0$ & $>12.0$ & $>12.0$ & $>12.0$ & $>12.0$ & $>12.0$ & $>12.0$ & $>12.0$ & $>12.0$ \\
\hline Glu141 & $<0.0$ & $<0.0$ & $<0.0$ & $<0.0$ & $<0.0$ & $<0.0$ & $<0.0$ & $<0.0$ & $<0.0$ & $<0.0$ & $<0.0$ & $<0.0$ & $<0.0$ & $<0.0$ & $<0.0$ & $<0.0$ \\
\hline Arg144 & $>12.0$ & $>12.0$ & $>12.0$ & $>12.0$ & $>12.0$ & $>12.0$ & $>12.0$ & $>12.0$ & $>12.0$ & $>12.0$ & $>12.0$ & $>12.0$ & $>12.0$ & $>12.0$ & $>12.0$ & $>12.0$ \\
\hline Arg145 & $>12.0$ & $>12.0$ & $>12.0$ & $>12.0$ & $>12.0$ & $>12.0$ & $>12.0$ & $>12.0$ & $>12.0$ & $>12.0$ & $>12.0$ & $>12.0$ & $>12.0$ & $>12.0$ & $>12.0$ & $>12.0$ \\
\hline
\end{tabular}




\begin{tabular}{|c|c|c|c|c|c|c|c|c|c|c|c|c|c|c|c|c|}
\hline His146 & $<0.0$ & $<0.0$ & $<0.0$ & 1.9 & $<0.0$ & $<0.0$ & 0.8 & 3.0 & $<0.0$ & $<0.0$ & $<0.0$ & 2.3 & $<0.0$ & $<0.0$ & 1.3 & 3.3 \\
\hline Tyr148 & $>12.0$ & $>12.0$ & $>12.0$ & $>12.0$ & $>12.0$ & $>12.0$ & $>12.0$ & $>12.0$ & $>12.0$ & $>12.0$ & $>12.0$ & $>12.0$ & $>12.0$ & $>12.0$ & $>12.0$ & $>12.0$ \\
\hline Tyr150 & $>12.0$ & $>12.0$ & $>12.0$ & 11.0 & $>12.0$ & $>12.0$ & $>12.0$ & $>12.0$ & $>12.0$ & $>12.0$ & $>12.0$ & $>12.0$ & $>12.0$ & $>12.0$ & $>12.0$ & $>12.0$ \\
\hline Glu153 & $<0.0$ & $<0.0$ & $<0.0$ & $<0.0$ & $<0.0$ & $<0.0$ & $<0.0$ & $<0.0$ & $<0.0$ & $<0.0$ & $<0.0$ & $<0.0$ & $<0.0$ & $<0.0$ & $<0.0$ & $<0.0$ \\
\hline Lys159 & $>12.0$ & $>12.0$ & $>12.0$ & $>12.0$ & 9.2 & 9.7 & 10.1 & 10.6 & 10.5 & 10.9 & 11.4 & $>12.0$ & 7.6 & 8.5 & 9.3 & 10.0 \\
\hline Arg160 & $>12.0$ & $>12.0$ & $>12.0$ & $>12.0$ & $>12.0$ & $>12.0$ & $>12.0$ & $>12.0$ & $>12.0$ & $>12.0$ & $>12.0$ & $>12.0$ & $>12.0$ & $>12.0$ & $>12.0$ & $>12.0$ \\
\hline Tyr161 & $>12.0$ & $>12.0$ & $>12.0$ & 11.6 & $>12.0$ & $>12.0$ & 11.7 & 10.4 & $>12.0$ & $>12.0$ & $>12.0$ & 11.6 & $>12.0$ & $>12.0$ & $>12.0$ & 10.9 \\
\hline Lys162 & $>12.0$ & $>12.0$ & $>12.0$ & $>12.0$ & $>12.0$ & $>12.0$ & $>12.0$ & $>12.0$ & $>12.0$ & $>12.0$ & $>12.0$ & $>12.0$ & $>12.0$ & $>12.0$ & $>12.0$ & $>12.0$ \\
\hline Glu167 & $<0.0$ & $<0.0$ & $<0.0$ & 0.3 & 3.2 & 3.3 & 3.4 & 3.4 & $<0.0$ & $<0.0$ & $<0.0$ & 0.2 & 1.7 & 2.2 & 2.6 & 2.9 \\
\hline Asp173 & $<0.0$ & $<0.0$ & $<0.0$ & $<0.0$ & 3.4 & 3.3 & 3.3 & 3.3 & $<0.0$ & $<0.0$ & $<0.0$ & $<0.0$ & 2.9 & 3.1 & 3.2 & 3.2 \\
\hline Lys174 & 10.7 & 11.2 & 11.6 & $>12.0$ & 11.2 & 11.3 & 11.5 & 11.6 & 10.8 & 11.3 & 11.8 & $>12.0$ & 11.2 & 11.3 & 11.4 & 11.5 \\
\hline Lys181 & $>12.0$ & $>12.0$ & $>12.0$ & $>12.0$ & 11.8 & $>12.0$ & $>12.0$ & $>12.0$ & $>12.0$ & $>12.0$ & $>12.0$ & $>12.0$ & 11.2 & 11.5 & 11.7 & $>12.0$ \\
\hline Asp183 & $<0.0$ & $<0.0$ & $<0.0$ & $<0.0$ & 0.1 & 0.6 & 1.0 & 1.3 & $<0.0$ & $<0.0$ & $<0.0$ & $<0.0$ & 0.7 & 0.8 & 0.8 & 0.9 \\
\hline Glu184 & 3.5 & 2.8 & 2.4 & 2.1 & 4.2 & 3.9 & 3.7 & 3.5 & 2.6 & 2.4 & 2.3 & 2.1 & 5.0 & 4.5 & 4.0 & 3.7 \\
\hline Arg186 & $>12.0$ & $>12.0$ & $>12.0$ & $>12.0$ & $>12.0$ & $>12.0$ & $>12.0$ & $>12.0$ & $>12.0$ & $>12.0$ & $>12.0$ & $>12.0$ & $>12.0$ & $>12.0$ & $>12.0$ & $>12.0$ \\
\hline Asp187 & $<0.0$ & $<0.0$ & $<0.0$ & $<0.0$ & 1.1 & 0.4 & $<0.0$ & $<0.0$ & $<0.0$ & $<0.0$ & $<0.0$ & $<0.0$ & $<0.0$ & $<0.0$ & $<0.0$ & $<0.0$ \\
\hline Glu188 & $<0.0$ & $<0.0$ & $<0.0$ & $<0.0$ & $<0.0$ & $<0.0$ & $<0.0$ & $<0.0$ & $<0.0$ & $<0.0$ & $<0.0$ & $<0.0$ & $<0.0$ & $<0.0$ & $<0.0$ & $<0.0$ \\
\hline Lys190 & 2.7 & 4.8 & 6.3 & 8.0 & 6.0 & 6.9 & 7.8 & 8.8 & 6.9 & 7.6 & 8.5 & 9.8 & 8.6 & 8.7 & 9.1 & 10.0 \\
\hline Lys195 & $>12.0$ & $>12.0$ & $>12.0$ & $>12.0$ & 11.4 & 11.6 & $>12.0$ & $>12.0$ & 8.1 & 9.5 & 11.3 & $>12.0$ & 8.6 & 11.2 & $>12.0$ & $>12.0$ \\
\hline Arg197 & $>12.0$ & $>12.0$ & $>12.0$ & $>12.0$ & $>12.0$ & $>12.0$ & $>12.0$ & $>12.0$ & $>12.0$ & $>12.0$ & $>12.0$ & $>12.0$ & $>12.0$ & $>12.0$ & $>12.0$ & $>12.0$ \\
\hline Lys199 & $<0.0$ & 2.3 & 6.4 & 8.9 & 0.5 & 4.2 & 7.1 & 9.4 & $<0.0$ & 3.0 & 6.4 & 9.5 & $<0.0$ & 0.5 & 6.2 & 9.7 \\
\hline Lys205 & 10.7 & 11.2 & 11.7 & $>12.0$ & $>12.0$ & $>12.0$ & $>12.0$ & $>12.0$ & $>12.0$ & $>12.0$ & $>12.0$ & $>12.0$ & $>12.0$ & $>12.0$ & $>12.0$ & $>12.0$ \\
\hline Glu208 & 3.1 & 3.1 & 2.9 & 2.9 & 2.5 & 2.5 & 2.6 & 2.7 & 1.9 & 2.0 & 2.1 & 2.3 & $<0.0$ & $<0.0$ & $<0.0$ & 0.5 \\
\hline Arg209 & $>12.0$ & $>12.0$ & $>12.0$ & $>12.0$ & $>12.0$ & $>12.0$ & $>12.0$ & $>12.0$ & $>12.0$ & $>12.0$ & $>12.0$ & $>12.0$ & $>12.0$ & $>12.0$ & $>12.0$ & $>12.0$ \\
\hline Lys212 & 9.8 & 10.3 & 10.8 & 11.3 & 10.6 & 10.8 & 11.1 & 11.3 & 11.6 & 11.6 & 11.6 & 11.7 & $>12.0$ & $>12.0$ & $>12.0$ & $>12.0$ \\
\hline Arg218 & $>12.0$ & $>12.0$ & $>12.0$ & $>12.0$ & $>12.0$ & $>12.0$ & $>12.0$ & $>12.0$ & $>12.0$ & $>12.0$ & $>12.0$ & $>12.0$ & $>12.0$ & $>12.0$ & $>12.0$ & $>12.0$ \\
\hline Arg222 & $>12.0$ & $>12.0$ & $>12.0$ & $>12.0$ & $>12.0$ & $>12.0$ & $>12.0$ & $>12.0$ & $>12.0$ & $>12.0$ & $>12.0$ & $>12.0$ & $>12.0$ & $>12.0$ & $>12.0$ & $>12.0$ \\
\hline Lys225 & 11.6 & 11.6 & 11.6 & 11.7 & 11.4 & 11.5 & 11.6 & 11.8 & $>12.0$ & $>12.0$ & $>12.0$ & $>12.0$ & $>12.0$ & $>12.0$ & $>12.0$ & $>12.0$ \\
\hline Glu227 & 3.5 & 3.6 & 3.9 & 4.1 & 3.5 & 3.7 & 3.9 & 4.0 & 3.7 & 3.9 & 4.1 & 4.2 & 3.6 & 3.8 & 3.9 & 4.0 \\
\hline Glu230 & 6.0 & 5.3 & 4.5 & 3.8 & 6.9 & 5.9 & 4.9 & 4.1 & 5.0 & 4.5 & 4.0 & 3.7 & 5.6 & 5.1 & 4.5 & 4.0 \\
\hline Lys233 & $>12.0$ & $>12.0$ & $>12.0$ & $>12.0$ & $>12.0$ & $>12.0$ & $>12.0$ & $>12.0$ & $>12.0$ & $>12.0$ & $>12.0$ & $>12.0$ & $>12.0$ & $>12.0$ & $>12.0$ & $>12.0$ \\
\hline Asp237 & 0.8 & 0.6 & 0.7 & 1.0 & 0.5 & 0.6 & 0.9 & 1.2 & $<0.0$ & $<0.0$ & 0.3 & 0.9 & $<0.0$ & $<0.0$ & 0.1 & 0.5 \\
\hline
\end{tabular}




\begin{tabular}{|c|c|c|c|c|c|c|c|c|c|c|c|c|c|c|c|c|}
\hline Lys240 & $>12.0$ & $>12.0$ & $>12.0$ & $>12.0$ & $>12.0$ & $>12.0$ & $>12.0$ & $>12.0$ & $>12.0$ & $>12.0$ & $>12.0$ & $>12.0$ & $>12.0$ & $>12.0$ & $>12.0$ & $>12.0$ \\
\hline His242 & $<0.0$ & $<0.0$ & $<0.0$ & $<0.0$ & $<0.0$ & $<0.0$ & $<0.0$ & $<0.0$ & $<0.0$ & $<0.0$ & $<0.0$ & $<0.0$ & $<0.0$ & $<0.0$ & $<0.0$ & $<0.0$ \\
\hline Glu244 & $>12.0$ & $>12.0$ & 8.6 & 5.8 & $>12.0$ & $>12.0$ & 8.5 & 5.4 & $>12.0$ & $>12.0$ & 8.5 & 5.3 & $>12.0$ & $>12.0$ & 10.5 & 6.1 \\
\hline His247 & 7.5 & 7.5 & 7.5 & 7.7 & 5.6 & 5.9 & 6.2 & 6.6 & 4.2 & 5.1 & 5.8 & 6.4 & $>12.0$ & $>12.0$ & $>12.0$ & 10.2 \\
\hline Asp249 & $<0.0$ & $<0.0$ & $<0.0$ & $<0.0$ & $<0.0$ & $<0.0$ & $<0.0$ & $<0.0$ & $<0.0$ & $<0.0$ & $<0.0$ & $<0.0$ & $<0.0$ & $<0.0$ & $<0.0$ & $<0.0$ \\
\hline Glu252 & 1.8 & 1.9 & 2.2 & 2.5 & 1.9 & 1.7 & 1.6 & 1.9 & 1.5 & 1.5 & 1.8 & 2.0 & $<0.0$ & $<0.0$ & $<0.0$ & $<0.0$ \\
\hline Asp255 & $<0.0$ & $<0.0$ & $<0.0$ & 0.7 & 0.6 & 0.8 & 1.2 & 1.8 & $<0.0$ & $<0.0$ & $<0.0$ & 0.5 & $<0.0$ & $<0.0$ & $<0.0$ & 0.6 \\
\hline Asp256 & 7.6 & 5.8 & 4.0 & 2.2 & 7.3 & 5.6 & 4.2 & 2.7 & 8.0 & 6.4 & 4.6 & 2.8 & 8.3 & 6.3 & 4.0 & 2.1 \\
\hline Arg257 & $>12.0$ & $>12.0$ & $>12.0$ & $>12.0$ & $>12.0$ & $>12.0$ & $>12.0$ & $>12.0$ & $>12.0$ & $>12.0$ & $>12.0$ & $>12.0$ & $>12.0$ & $>12.0$ & $>12.0$ & $>12.0$ \\
\hline Asp259 & 3.7 & 3.5 & 3.5 & 3.4 & 4.0 & 3.7 & 3.7 & 3.5 & 4.1 & 3.7 & 3.7 & 3.6 & 3.1 & 2.9 & 2.9 & 3.0 \\
\hline Lys262 & $>12.0$ & $>12.0$ & $>12.0$ & $>12.0$ & $>12.0$ & $>12.0$ & $>12.0$ & $>12.0$ & $>12.0$ & $>12.0$ & $>12.0$ & $>12.0$ & $>12.0$ & $>12.0$ & $>12.0$ & $>12.0$ \\
\hline Tyr263 & $>12.0$ & $>12.0$ & $>12.0$ & $>12.0$ & $>12.0$ & $>12.0$ & $>12.0$ & $>12.0$ & $>12.0$ & $>12.0$ & $>12.0$ & $>12.0$ & $>12.0$ & $>12.0$ & $>12.0$ & $>12.0$ \\
\hline Glu266 & 4.0 & 3.9 & 3.9 & 3.8 & 4.8 & 4.4 & 4.1 & 3.8 & 2.9 & 3.1 & 3.2 & 3.3 & 5.2 & 4.7 & 4.3 & 3.9 \\
\hline Asp269 & 3.6 & 3.7 & 3.7 & 3.7 & 3.0 & 3.1 & 3.1 & 3.2 & 3.8 & 3.8 & 3.8 & 3.8 & 3.8 & 3.8 & 3.8 & 3.7 \\
\hline Lys274 & $>12.0$ & $>12.0$ & $>12.0$ & $>12.0$ & $>12.0$ & $>12.0$ & $>12.0$ & $>12.0$ & $>12.0$ & $>12.0$ & $>12.0$ & $>12.0$ & $>12.0$ & $>12.0$ & $>12.0$ & $>12.0$ \\
\hline Lys276 & 11.5 & 11.5 & 11.7 & 11.8 & $>12.0$ & 11.8 & 11.6 & 11.6 & 11.6 & 11.7 & $>12.0$ & $>12.0$ & 10.7 & 10.8 & 11.0 & 11.1 \\
\hline Glu277 & 1.2 & 1.5 & 1.8 & 2.2 & 6.0 & 5.2 & 4.5 & 3.9 & 0.5 & 0.9 & 1.4 & 2.0 & 2.8 & 3.0 & 3.1 & 3.3 \\
\hline Glu280 & 4.2 & 4.1 & 4.0 & 3.9 & 4.3 & 4.2 & 4.1 & 4.0 & 4.5 & 4.3 & 4.1 & 3.8 & 4.3 & 4.2 & 4.1 & 4.0 \\
\hline Lys281 & 11.7 & 11.7 & 11.8 & $>12.0$ & $>12.0$ & $>12.0$ & $>12.0$ & $>12.0$ & 10.3 & 11.2 & 11.8 & $>12.0$ & $>12.0$ & $>12.0$ & $>12.0$ & $>12.0$ \\
\hline Glu285 & $<0.0$ & $<0.0$ & $<0.0$ & $<0.0$ & $<0.0$ & $<0.0$ & 0.3 & 1.0 & $<0.0$ & $<0.0$ & $<0.0$ & $<0.0$ & 1.1 & 1.0 & 1.0 & 1.1 \\
\hline Lys286 & 10.3 & 10.7 & 11.1 & 11.5 & 9.8 & 10.3 & 10.8 & 11.3 & 10.9 & 11.2 & 11.6 & $>12.0$ & 9.2 & 10.1 & 10.7 & 11.3 \\
\hline His288 & 6.7 & 6.6 & 6.3 & 6.4 & 8.0 & 7.5 & 7.1 & 7.0 & $>12.0$ & 10.9 & 8.4 & 7.6 & 8.8 & 7.5 & 7.1 & 7.0 \\
\hline Glu292 & $<0.0$ & $<0.0$ & $<0.0$ & $<0.0$ & $<0.0$ & $<0.0$ & $<0.0$ & $<0.0$ & $<0.0$ & $<0.0$ & $<0.0$ & $<0.0$ & $<0.0$ & $<0.0$ & $<0.0$ & 0.0 \\
\hline Glu294 & 2.8 & 2.3 & 1.8 & 1.8 & 3.6 & 3.3 & 3.2 & 3.1 & 4.8 & 4.2 & 3.5 & 2.8 & 4.2 & 3.7 & 2.9 & 2.7 \\
\hline Asp296 & $<0.0$ & $<0.0$ & $<0.0$ & 0.6 & $<0.0$ & $<0.0$ & 0.5 & 1.2 & $<0.0$ & $<0.0$ & $<0.0$ & 0.1 & 0.3 & 0.5 & 0.7 & 1.0 \\
\hline Glu297 & 2.5 & 2.7 & 2.9 & 3.0 & 4.8 & 4.5 & 4.3 & 4.1 & 2.6 & 2.8 & 2.9 & 3.0 & 3.2 & 3.3 & 3.4 & 3.3 \\
\hline Asp301 & 3.6 & 3.6 & 3.6 & 3.7 & 3.8 & 3.8 & 3.8 & 3.8 & 3.7 & 3.7 & 3.7 & 3.7 & 3.7 & 3.8 & 3.8 & 3.8 \\
\hline Asp308 & 5.1 & 4.7 & 4.2 & 3.8 & 3.5 & 3.5 & 3.4 & 3.4 & 4.1 & 3.9 & 3.7 & 3.5 & 3.8 & 3.7 & 3.7 & 3.6 \\
\hline Glu311 & 4.1 & 4.1 & 4.1 & 4.1 & 4.1 & 4.1 & 4.1 & 4.0 & 4.9 & 4.6 & 4.4 & 4.2 & 4.5 & 4.3 & 4.2 & 4.0 \\
\hline Lys313 & 11.0 & 11.0 & 11.0 & 11.1 & 11.1 & 11.1 & 11.0 & 11.0 & 11.3 & 11.2 & 11.2 & 11.2 & 11.3 & 11.2 & 11.2 & 11.2 \\
\hline Asp314 & 2.2 & 2.4 & 2.6 & 2.7 & 2.3 & 2.5 & 2.7 & 2.9 & 2.3 & 2.4 & 2.6 & 2.7 & 6.6 & 5.4 & 4.2 & 3.1 \\
\hline Lys317 & 11.4 & 11.4 & 11.4 & 11.4 & 11.4 & 11.4 & 11.4 & 11.4 & 11.4 & 11.4 & 11.5 & 11.5 & $>12.0$ & $>12.0$ & $>12.0$ & $>12.0$ \\
\hline
\end{tabular}




\begin{tabular}{|c|c|c|c|c|c|c|c|c|c|c|c|c|c|c|c|c|}
\hline Tyr319 & $>12.0$ & $>12.0$ & $>12.0$ & $>12.0$ & $>12.0$ & $>12.0$ & $>12.0$ & $>12.0$ & $>12.0$ & $>12.0$ & $>12.0$ & $>12.0$ & $>12.0$ & $>12.0$ & $>12.0$ & $>12.0$ \\
\hline Glu321 & 4.5 & 4.3 & 4.2 & 4.1 & 5.2 & 5.0 & 4.7 & 4.5 & 4.9 & 4.7 & 4.5 & 4.4 & 4.8 & 4.6 & 4.3 & 4.1 \\
\hline Lys323 & $>12.0$ & $>12.0$ & $>12.0$ & $>12.0$ & $>12.0$ & $>12.0$ & $>12.0$ & $>12.0$ & $>12.0$ & $>12.0$ & $>12.0$ & $>12.0$ & $>12.0$ & $>12.0$ & $>12.0$ & $>12.0$ \\
\hline Asp324 & 1.5 & 1.6 & 1.7 & 2.0 & 0.6 & 0.8 & 1.1 & 1.4 & 0.5 & 0.7 & 1.0 & 1.3 & $<0.0$ & $<0.0$ & $<0.0$ & $<0.0$ \\
\hline Tyr332 & $>12.0$ & $>12.0$ & $>12.0$ & $>12.0$ & $>12.0$ & $>12.0$ & $>12.0$ & $>12.0$ & $>12.0$ & $>12.0$ & $>12.0$ & $>12.0$ & $>12.0$ & $>12.0$ & $>12.0$ & $>12.0$ \\
\hline Glu333 & 2.1 & 2.2 & 2.2 & 2.4 & 0.5 & 1.0 & 1.5 & 2.0 & 2.0 & 2.1 & 2.3 & 2.5 & $<0.0$ & $<0.0$ & 0.8 & 1.5 \\
\hline Tyr334 & $>12.0$ & $>12.0$ & $>12.0$ & 11.7 & $>12.0$ & $>12.0$ & $>12.0$ & 11.4 & $>12.0$ & $>12.0$ & $>12.0$ & $>12.0$ & $>12.0$ & $>12.0$ & $>12.0$ & 11.4 \\
\hline Arg336 & $>12.0$ & $>12.0$ & $>12.0$ & $>12.0$ & $>12.0$ & $>12.0$ & $>12.0$ & $>12.0$ & $>12.0$ & $>12.0$ & $>12.0$ & $>12.0$ & $>12.0$ & $>12.0$ & $>12.0$ & $>12.0$ \\
\hline Arg337 & $>12.0$ & $>12.0$ & $>12.0$ & $>12.0$ & $>12.0$ & $>12.0$ & $>12.0$ & $>12.0$ & $>12.0$ & $>12.0$ & $>12.0$ & $>12.0$ & $>12.0$ & $>12.0$ & $>12.0$ & $>12.0$ \\
\hline His338 & 8.2 & 7.9 & 7.7 & 7.5 & 7.6 & 7.4 & 7.4 & 7.3 & 6.8 & 6.9 & 6.9 & 6.9 & 6.8 & 6.9 & 6.9 & 7.0 \\
\hline Asp340 & 0.1 & 0.4 & 0.7 & 1.0 & 1.1 & 1.1 & 1.2 & 1.3 & 1.3 & 1.2 & 1.2 & 1.3 & 0.8 & 0.9 & 1.1 & 1.3 \\
\hline Tyr341 & $>12.0$ & $>12.0$ & $>12.0$ & $>12.0$ & $>12.0$ & $>12.0$ & $>12.0$ & $>12.0$ & $>12.0$ & $>12.0$ & $>12.0$ & $>12.0$ & $>12.0$ & $>12.0$ & $>12.0$ & $>12.0$ \\
\hline Arg348 & $>12.0$ & $>12.0$ & $>12.0$ & $>12.0$ & $>12.0$ & $>12.0$ & $>12.0$ & $>12.0$ & $>12.0$ & $>12.0$ & $>12.0$ & $>12.0$ & $>12.0$ & $>12.0$ & $>12.0$ & $>12.0$ \\
\hline Lys351 & 11.1 & 11.1 & 11.2 & 11.3 & 8.7 & 9.2 & 9.7 & 10.2 & 10.2 & 10.4 & 10.6 & 10.8 & 10.6 & 10.8 & 11.2 & 11.6 \\
\hline Tyr353 & $>12.0$ & $>12.0$ & $>12.0$ & $>12.0$ & $>12.0$ & $>12.0$ & $>12.0$ & $>12.0$ & $>12.0$ & $>12.0$ & $>12.0$ & $>12.0$ & $>12.0$ & $>12.0$ & $>12.0$ & $>12.0$ \\
\hline Glu354 & 0.5 & 0.8 & 1.2 & 1.7 & 3.7 & 3.3 & 2.9 & 2.7 & 2.9 & 2.2 & 1.9 & 1.8 & 1.8 & 1.5 & 1.1 & 1.0 \\
\hline Glu358 & 3.9 & 4.0 & 4.0 & 3.9 & 1.8 & 2.4 & 2.8 & 3.1 & 3.2 & 3.5 & 3.6 & 3.6 & $<0.0$ & $<0.0$ & 0.1 & 1.1 \\
\hline Lys359 & 10.8 & 10.8 & 10.9 & 10.9 & 11.0 & 11.1 & 11.1 & 11.1 & 10.7 & 10.8 & 10.9 & 11.0 & 11.6 & 11.6 & 11.7 & 11.7 \\
\hline Asp365 & 2.9 & 3.1 & 3.2 & 3.3 & 3.1 & 3.1 & 3.1 & 3.1 & 3.1 & 3.3 & 3.4 & 3.5 & 2.1 & 2.2 & 2.3 & 2.3 \\
\hline His367 & 6.2 & 6.5 & 6.8 & 6.9 & 8.5 & 8.2 & 7.9 & 7.7 & 6.5 & 6.9 & 7.1 & 7.3 & 7.9 & 7.8 & 7.6 & 7.5 \\
\hline Glu368 & 3.8 & 3.9 & 4.0 & 4.1 & 4.0 & 4.0 & 3.9 & 3.9 & 4.7 & 4.5 & 4.4 & 4.4 & 3.6 & 3.7 & 3.8 & 3.9 \\
\hline Tyr370 & $>12.0$ & $>12.0$ & $>12.0$ & $>12.0$ & $>12.0$ & $>12.0$ & $>12.0$ & $>12.0$ & $>12.0$ & $>12.0$ & $>12.0$ & $>12.0$ & $>12.0$ & $>12.0$ & $>12.0$ & $>12.0$ \\
\hline Lys372 & 10.9 & 11.0 & 11.1 & 11.2 & 11.8 & $>12.0$ & $>12.0$ & $>12.0$ & 10.8 & 10.9 & 11.0 & 11.1 & 11.3 & 11.3 & 11.4 & 11.5 \\
\hline Asp375 & 3.0 & 3.1 & 3.1 & 3.1 & 2.7 & 2.7 & 2.7 & 2.8 & 3.4 & 3.3 & 3.2 & 3.1 & 2.8 & 2.8 & 2.9 & 2.9 \\
\hline Glu376 & 4.8 & 4.5 & 4.2 & 3.9 & 6.6 & 5.8 & 5.1 & 4.5 & 6.0 & 5.4 & 4.8 & 4.4 & 5.3 & 4.9 & 4.5 & 4.2 \\
\hline Lys378 & 11.2 & 11.3 & 11.4 & 11.5 & 11.1 & 11.2 & 11.5 & $>12.0$ & 11.1 & 11.1 & 11.2 & 11.4 & 10.8 & 10.9 & 11.0 & 11.1 \\
\hline Glu382 & 4.6 & 4.4 & 4.1 & 3.9 & 4.0 & 3.7 & 3.5 & 3.4 & 4.9 & 4.5 & 4.2 & 3.9 & 4.8 & 4.5 & 4.3 & 4.1 \\
\hline Glu383 & $<0.0$ & $<0.0$ & 0.5 & 1.0 & $<0.0$ & $<0.0$ & $<0.0$ & $<0.0$ & 0.5 & 0.8 & 1.0 & 1.3 & $<0.0$ & $<0.0$ & 0.3 & 0.9 \\
\hline Lys389 & 10.9 & 11.0 & 11.0 & 11.2 & 11.7 & 11.8 & $>12.0$ & $>12.0$ & 11.1 & 11.2 & 11.3 & 11.5 & 11.4 & 11.5 & 11.6 & 11.7 \\
\hline Glu393 & 3.7 & 3.5 & 3.2 & 3.0 & 2.9 & 2.7 & 2.6 & 2.6 & 3.4 & 3.4 & 3.4 & 3.3 & 2.9 & 3.0 & 3.0 & 3.0 \\
\hline Glu396 & 3.6 & 3.2 & 2.6 & 2.2 & 4.9 & 4.5 & 4.1 & 3.7 & 3.6 & 3.0 & 2.3 & 1.9 & 4.9 & 4.4 & 4.0 & 3.6 \\
\hline Glu400 & $<0.0$ & $<0.0$ & $<0.0$ & $<0.0$ & 1.2 & 1.1 & 1.0 & 1.0 & $<0.0$ & $<0.0$ & $<0.0$ & $<0.0$ & $<0.0$ & $<0.0$ & $<0.0$ & 0.2 \\
\hline
\end{tabular}




\begin{tabular}{|c|c|c|c|c|c|c|c|c|c|c|c|c|c|c|c|c|}
\hline Tyr401 & $>12.0$ & $>12.0$ & $>12.0$ & 10.8 & $>12.0$ & $>12.0$ & 10.5 & 8.4 & $>12.0$ & $>12.0$ & $>12.0$ & 9.4 & $>12.0$ & $>12.0$ & $>12.0$ & 9.6 \\
\hline Lys402 & 11.7 & 11.7 & 11.7 & $>12.0$ & 10.6 & 10.7 & 10.9 & 11.1 & 11.7 & 11.7 & 11.7 & $>12.0$ & 10.5 & 10.8 & 10.9 & 11.2 \\
\hline Arg410 & $>12.0$ & $>12.0$ & $>12.0$ & $>12.0$ & $>12.0$ & $>12.0$ & $>12.0$ & $>12.0$ & $>12.0$ & $>12.0$ & $>12.0$ & $>12.0$ & $>12.0$ & $>12.0$ & $>12.0$ & $>12.0$ \\
\hline Tyr411 & $>12.0$ & $>12.0$ & 9.5 & 8.8 & $>12.0$ & $>12.0$ & 9.0 & 8.3 & $>12.0$ & 7.9 & 7.7 & 8.0 & $>12.0$ & $>12.0$ & 9.8 & 8.8 \\
\hline Lys413 & 10.5 & 10.3 & 10.2 & 10.6 & $>12.0$ & $>12.0$ & $>12.0$ & $>12.0$ & 9.8 & 10.1 & 10.5 & 10.9 & 9.8 & 10.3 & 10.5 & 10.9 \\
\hline Lys414 & 7.6 & 8.6 & 11.0 & $>12.0$ & 6.7 & 8.2 & $>12.0$ & $>12.0$ & 9.8 & 10.1 & 10.5 & 10.9 & 9.8 & 10.3 & 10.5 & 10.9 \\
\hline Glu425 & $<0.0$ & $<0.0$ & $<0.0$ & $<0.0$ & $<0.0$ & $<0.0$ & $<0.0$ & $<0.0$ & $<0.0$ & $<0.0$ & $<0.0$ & $<0.0$ & $<0.0$ & $<0.0$ & $<0.0$ & $<0.0$ \\
\hline Arg428 & $>12.0$ & $>12.0$ & $>12.0$ & $>12.0$ & $>12.0$ & $>12.0$ & $>12.0$ & $>12.0$ & $>12.0$ & $>12.0$ & $>12.0$ & $>12.0$ & $>12.0$ & $>12.0$ & $>12.0$ & $>12.0$ \\
\hline Lys432 & 8.8 & 9.6 & 10.4 & $>12.0$ & 8.8 & 9.0 & 9.5 & 10.4 & 4.9 & 6.2 & 7.7 & 10.3 & 7.5 & 8.3 & 9.5 & 11.3 \\
\hline Lys436 & 10.8 & 11.0 & 11.3 & $>12.0$ & 7.9 & 8.8 & 9.7 & 11.5 & 10.6 & 11.1 & 11.8 & $>12.0$ & 10.2 & 10.5 & 11.1 & $>12.0$ \\
\hline Lys439 & $>12.0$ & $>12.0$ & $>12.0$ & $>12.0$ & 11.1 & 11.2 & 11.2 & 11.2 & 11.3 & 11.4 & 11.3 & 11.3 & 11.1 & 11.1 & 11.1 & 11.1 \\
\hline His440 & $<0.0$ & 1.0 & 2.9 & 4.4 & 0.9 & 2.2 & 3.5 & 4.6 & $<0.0$ & $<0.0$ & 1.9 & 3.9 & 0.6 & 2.0 & 3.4 & 4.7 \\
\hline Glus442 & 3.2 & 3.3 & 3.3 & 3.2 & 2.9 & 3.0 & 3.1 & 3.1 & 2.7 & 2.7 & 2.6 & 2.4 & 2.9 & 2.9 & 2.9 & 3.0 \\
\hline Lys444 & 9.5 & 9.9 & 10.4 & 10.8 & 9.5 & 9.9 & 10.2 & 10.6 & 9.3 & 9.6 & 10.0 & 10.5 & 9.4 & 9.9 & 10.3 & 10.7 \\
\hline Arg445 & $>12.0$ & $>12.0$ & $>12.0$ & $>12.0$ & $>12.0$ & $>12.0$ & $>12.0$ & $>12.0$ & $>12.0$ & $>12.0$ & $>12.0$ & $>12.0$ & $>12.0$ & $>12.0$ & $>12.0$ & $>12.0$ \\
\hline Glu450 & $<0.0$ & $<0.0$ & $<0.0$ & $<0.0$ & $<0.0$ & $<0.0$ & $<0.0$ & $<0.0$ & $<0.0$ & $<0.0$ & $<0.0$ & $<0.0$ & $<0.0$ & $<0.0$ & $<0.0$ & $<0.0$ \\
\hline Asp451 & $<0.0$ & $<0.0$ & $<0.0$ & $<0.0$ & $<0.0$ & $<0.0$ & $<0.0$ & $<0.0$ & $<0.0$ & $<0.0$ & $<0.0$ & $<0.0$ & $<0.0$ & $<0.0$ & $<0.0$ & $<0.0$ \\
\hline Tyr452 & $>12.0$ & $>12.0$ & $>12.0$ & $>12.0$ & $>12.0$ & $>12.0$ & $>12.0$ & $>12.0$ & $>12.0$ & $>12.0$ & $>12.0$ & 9.3 & $>12.0$ & $>12.0$ & $>12.0$ & 10.6 \\
\hline His464 & $<0.0$ & $<0.0$ & 3.2 & 5.2 & $<0.0$ & $<0.0$ & 1.8 & 4.7 & $<0.0$ & $<0.0$ & 2.4 & 5.0 & $<0.0$ & $<0.0$ & 0.4 & 4.6 \\
\hline Glu465 & 2.2 & 2.0 & 1.0 & 1.2 & 1.7 & 1.7 & 1.3 & 1.2 & 2.7 & 2.4 & 1.7 & 1.5 & 2.6 & 2.0 & 0.7 & 0.1 \\
\hline Lys466 & $>12.0$ & $>12.0$ & $>12.0$ & $>12.0$ & $>12.0$ & $>12.0$ & $>12.0$ & $>12.0$ & $>12.0$ & $>12.0$ & $>12.0$ & $>12.0$ & $>12.0$ & $>12.0$ & $>12.0$ & $>12.0$ \\
\hline Asp471 & 2.4 & 2.6 & 2.5 & 2.6 & 2.2 & 2.5 & 2.5 & 2.3 & 2.2 & 2.4 & 2.4 & 2.4 & 2.0 & 2.2 & 2.4 & 2.2 \\
\hline Arg472 & $>12.0$ & $>12.0$ & $>12.0$ & $>12.0$ & $>12.0$ & $>12.0$ & $>12.0$ & $>12.0$ & $>12.0$ & $>12.0$ & $>12.0$ & $>12.0$ & $>12.0$ & $>12.0$ & $>12.0$ & $>12.0$ \\
\hline Lys475 & 11.0 & 11.0 & 11.0 & 11.1 & $>12.0$ & $>12.0$ & $>12.0$ & $>12.0$ & 8.6 & 9.5 & 10.1 & 10.7 & 11.7 & 11.5 & 11.6 & 11.6 \\
\hline Glu479 & 3.9 & 3.6 & 3.4 & 3.2 & 1.7 & 1.9 & 2.1 & 2.3 & 3.9 & 3.7 & 3.5 & 3.3 & 2.2 & 2.3 & 2.3 & 2.2 \\
\hline Arg484 & $>12.0$ & $>12.0$ & $>12.0$ & $>12.0$ & $>12.0$ & $>12.0$ & $>12.0$ & $>12.0$ & $>12.0$ & $>12.0$ & $>12.0$ & $>12.0$ & $>12.0$ & $>12.0$ & $>12.0$ & $>12.0$ \\
\hline Arg485 & $>12.0$ & $>12.0$ & $>12.0$ & $>12.0$ & $>12.0$ & $>12.0$ & $>12.0$ & $>12.0$ & $>12.0$ & $>12.0$ & $>12.0$ & $>12.0$ & $>12.0$ & $>12.0$ & $>12.0$ & $>12.0$ \\
\hline Glu492 & 2.9 & 3.0 & 3.1 & 3.2 & $<0.0$ & $<0.0$ & $<0.0$ & $<0.0$ & 2.7 & 2.8 & 2.9 & 3.0 & 3.2 & 3.2 & 3.3 & 3.4 \\
\hline Asp494 & 1.1 & 1.3 & 1.4 & 1.6 & $<0.0$ & $<0.0$ & $<0.0$ & $<0.0$ & 0.9 & 1.2 & 1.3 & 1.5 & 0.6 & 1.0 & 1.3 & 1.5 \\
\hline Glu495 & 4.5 & 4.4 & 4.3 & 4.2 & 1.6 & 1.6 & 1.5 & 1.5 & 4.7 & 4.5 & 4.2 & 4.0 & 4.7 & 4.5 & 4.3 & 4.1 \\
\hline Tyr497 & $>12.0$ & $>12.0$ & $>12.0$ & $>12.0$ & $>12.0$ & $>12.0$ & $>12.0$ & 11.5 & $>12.0$ & $>12.0$ & $>12.0$ & 11.8 & $>12.0$ & $>12.0$ & $>12.0$ & 11.3 \\
\hline Lys500 & 11.0 & 11.0 & 11.0 & 11.0 & $>12.0$ & $>12.0$ & $>12.0$ & $>12.0$ & 11.0 & 11.0 & 11.0 & 11.2 & $>12.0$ & $>12.0$ & $>12.0$ & $>12.0$ \\
\hline
\end{tabular}




\begin{tabular}{|c|c|c|c|c|c|c|c|c|c|c|c|c|c|c|c|c|}
\hline Glu501 & 3.6 & 3.7 & 3.7 & 3.8 & 3.2 & 3.3 & 3.4 & 3.4 & 4.3 & 4.2 & 3.9 & 3.8 & 3.6 & 3.7 & 3.8 & 3.8 \\
\hline Glu505 & 3.7 & 3.7 & 3.7 & 3.7 & 2.7 & 3.0 & 3.2 & 3.4 & 3.7 & 3.6 & 3.6 & 3.7 & 3.7 & 3.7 & 3.8 & 3.8 \\
\hline His510 & 7.6 & 7.6 & 7.7 & 7.7 & $<0.0$ & 1.4 & 4.8 & 5.7 & 6.1 & 6.6 & 6.9 & 7.1 & 5.9 & 6.2 & 6.4 & 6.6 \\
\hline Asp512 & 0.5 & 0.9 & 1.3 & 1.5 & 5.1 & 4.5 & 2.6 & 2.4 & 1.4 & 1.5 & 1.7 & 1.8 & 1.1 & 1.3 & 1.4 & 1.6 \\
\hline Glu518 & 1.7 & 0.8 & 0.2 & $<0.0$ & 2.6 & 2.4 & 2.2 & 2.1 & $<0.0$ & $<0.0$ & $<0.0$ & $<0.0$ & 2.5 & 2.1 & 1.8 & 1.6 \\
\hline Lys519 & 8.9 & 9.0 & 9.3 & 9.7 & 11.0 & 10.8 & 10.8 & 10.8 & 11.3 & 11.4 & 11.5 & $>12.0$ & 5.7 & 7.2 & 8.4 & 9.5 \\
\hline Glu520 & $<0.0$ & $<0.0$ & $<0.0$ & $<0.0$ & 0.5 & 0.8 & 1.2 & 1.5 & $<0.0$ & $<0.0$ & $<0.0$ & $<0.0$ & 0.6 & 0.8 & 0.9 & 1.1 \\
\hline Arg521 & $>12.0$ & $>12.0$ & $>12.0$ & $>12.0$ & $>12.0$ & $>12.0$ & $>12.0$ & $>12.0$ & $>12.0$ & $>12.0$ & $>12.0$ & $>12.0$ & $>12.0$ & $>12.0$ & $>12.0$ & $>12.0$ \\
\hline Lys524 & 9.3 & 9.8 & 10.3 & 10.9 & 8.2 & 8.9 & 9.6 & 10.4 & 9.9 & 10.4 & 10.8 & 11.2 & 9.3 & 9.8 & 10.2 & 10.6 \\
\hline Lys525 & 8.5 & 10.1 & 11.7 & $>12.0$ & 7.0 & 8.0 & 9.7 & 11.6 & 7.0 & 8.4 & 9.7 & $>12.0$ & 7.5 & 8.6 & 9.7 & $>12.0$ \\
\hline Glu531 & 0.7 & 0.9 & 1.1 & 1.4 & $<0.0$ & $<0.0$ & 0.0 & 0.6 & 0.3 & $<0.0$ & $<0.0$ & 0.5 & $<0.0$ & $<0.0$ & $<0.0$ & 0.2 \\
\hline Lys534 & $>12.0$ & $>12.0$ & $>12.0$ & $>12.0$ & $>12.0$ & $>12.0$ & $>12.0$ & $>12.0$ & $>12.0$ & $>12.0$ & $>12.0$ & $>12.0$ & $>12.0$ & $>12.0$ & $>12.0$ & $>12.0$ \\
\hline His535 & 9.7 & 8.3 & 7.9 & 7.7 & 4.3 & 5.1 & 5.7 & 6.0 & 2.1 & 3.2 & 5.0 & 5.9 & 5.0 & 5.4 & 5.7 & 6.4 \\
\hline Lys536 & 7.8 & 10.2 & 11.3 & $>12.0$ & $>12.0$ & $>12.0$ & $>12.0$ & $>12.0$ & 1.3 & 7.3 & 8.9 & 10.6 & 10.4 & 11.2 & 11.3 & 11.3 \\
\hline Lys538 & 10.7 & 10.8 & 10.8 & 10.8 & 8.4 & 9.1 & 9.9 & 10.8 & 11.1 & 11.0 & 11.0 & 10.9 & 10.5 & 10.6 & 10.6 & 10.6 \\
\hline Lys541 & 10.1 & 10.1 & 10.2 & 10.3 & 7.7 & 8.2 & 8.8 & 9.5 & 10.6 & 10.6 & 10.7 & 10.7 & 11.6 & 11.2 & 10.8 & 10.6 \\
\hline Glu542 & 2.5 & 2.7 & 3.0 & 3.1 & 1.2 & 1.4 & 1.6 & 1.8 & 3.1 & 3.0 & 3.1 & 3.2 & 2.3 & 2.3 & 2.3 & 2.3 \\
\hline Lys545 & 9.2 & 9.5 & 9.9 & 10.4 & 11.5 & 11.5 & 11.6 & 11.7 & 10.1 & 10.2 & 10.4 & 10.6 & 9.1 & 9.7 & 10.4 & 10.9 \\
\hline Asp549 & 1.7 & 1.6 & 1.6 & 1.7 & 2.6 & 2.3 & 2.1 & 2.0 & 0.2 & 0.4 & 0.7 & 1.1 & 2.0 & 2.0 & 2.1 & 2.1 \\
\hline Asp550 & 6.1 & 5.2 & 4.3 & 3.5 & 5.4 & 4.9 & 4.4 & 4.0 & 6.5 & 5.0 & 3.9 & 3.0 & $>12.0$ & 8.8 & 6.7 & 5.0 \\
\hline Glu556 & 3.1 & 2.8 & 2.5 & 2.3 & 2.9 & 2.8 & 2.8 & 2.9 & 0.8 & 1.0 & 1.3 & 1.5 & 3.1 & 3.2 & 3.2 & 3.2 \\
\hline Lys557 & 11.8 & 11.8 & $>12.0$ & $>12.0$ & 11.3 & 11.4 & 11.4 & 11.4 & 9.8 & 10.7 & 11.3 & 11.8 & $>12.0$ & $>12.0$ & $>12.0$ & $>12.0$ \\
\hline Lys560 & $>12.0$ & $>12.0$ & $>12.0$ & $>12.0$ & 10.5 & 10.6 & 10.7 & 10.8 & $>12.0$ & $>12.0$ & $>12.0$ & $>12.0$ & 10.9 & 10.9 & 10.9 & 10.9 \\
\hline Asp562 & 3.3 & 3.3 & 3.4 & 3.4 & 4.1 & 4.0 & 3.9 & 3.9 & 3.9 & 3.8 & 3.7 & 3.5 & 3.5 & 3.6 & 3.6 & 3.7 \\
\hline Asp563 & 4.2 & 3.0 & 2.7 & 2.5 & 2.3 & 2.6 & 2.8 & 3.0 & $<0.0$ & $<0.0$ & 0.8 & 1.4 & 10.4 & 8.3 & 6.6 & 5.1 \\
\hline Lys564 & 11.5 & 11.5 & 11.4 & 11.4 & 11.4 & 11.5 & 11.5 & 11.5 & 11.1 & 11.2 & 11.2 & 11.2 & $>12.0$ & $>12.0$ & $>12.0$ & $>12.0$ \\
\hline Glu565 & 3.5 & 3.7 & 3.8 & 3.9 & 4.3 & 4.3 & 4.2 & 4.1 & 3.4 & 3.5 & 3.6 & 3.6 & 3.1 & 3.3 & 3.5 & 3.6 \\
\hline Glu570 & 3.8 & 3.9 & 3.9 & 3.9 & 3.4 & 3.5 & 3.5 & 3.6 & 4.8 & 4.6 & 4.5 & 4.3 & 4.4 & 4.3 & 4.3 & 4.3 \\
\hline Glu571 & 4.7 & 4.4 & 4.2 & 4.0 & 3.2 & 3.3 & 3.2 & 3.2 & 3.0 & 3.2 & 3.4 & 3.6 & 6.3 & 5.4 & 4.5 & 3.9 \\
\hline Lys573 & 10.9 & 10.9 & 10.9 & 11.0 & 8.0 & 9.0 & 9.8 & 10.5 & 9.8 & 10.1 & 10.4 & 10.6 & 10.7 & 10.7 & 10.8 & 10.8 \\
\hline Lys574 & 10.7 & 10.7 & 10.8 & 10.8 & 10.5 & 10.7 & 10.9 & 11.1 & 10.6 & 10.6 & 10.7 & 10.8 & 10.7 & 11.2 & 11.2 & 11.2 \\
\hline
\end{tabular}


Figure S1. Time evolution along the STD-a trajectory of the root mean squared deviation (RMSD, in $\AA$ ) computed for the heavy backbone atoms in the three domains, six subdomains, and three subdomain connecting loops with respect to the 3JRY crystal structure (molecule A). Average values and standard deviations for the last 200 ns are included.
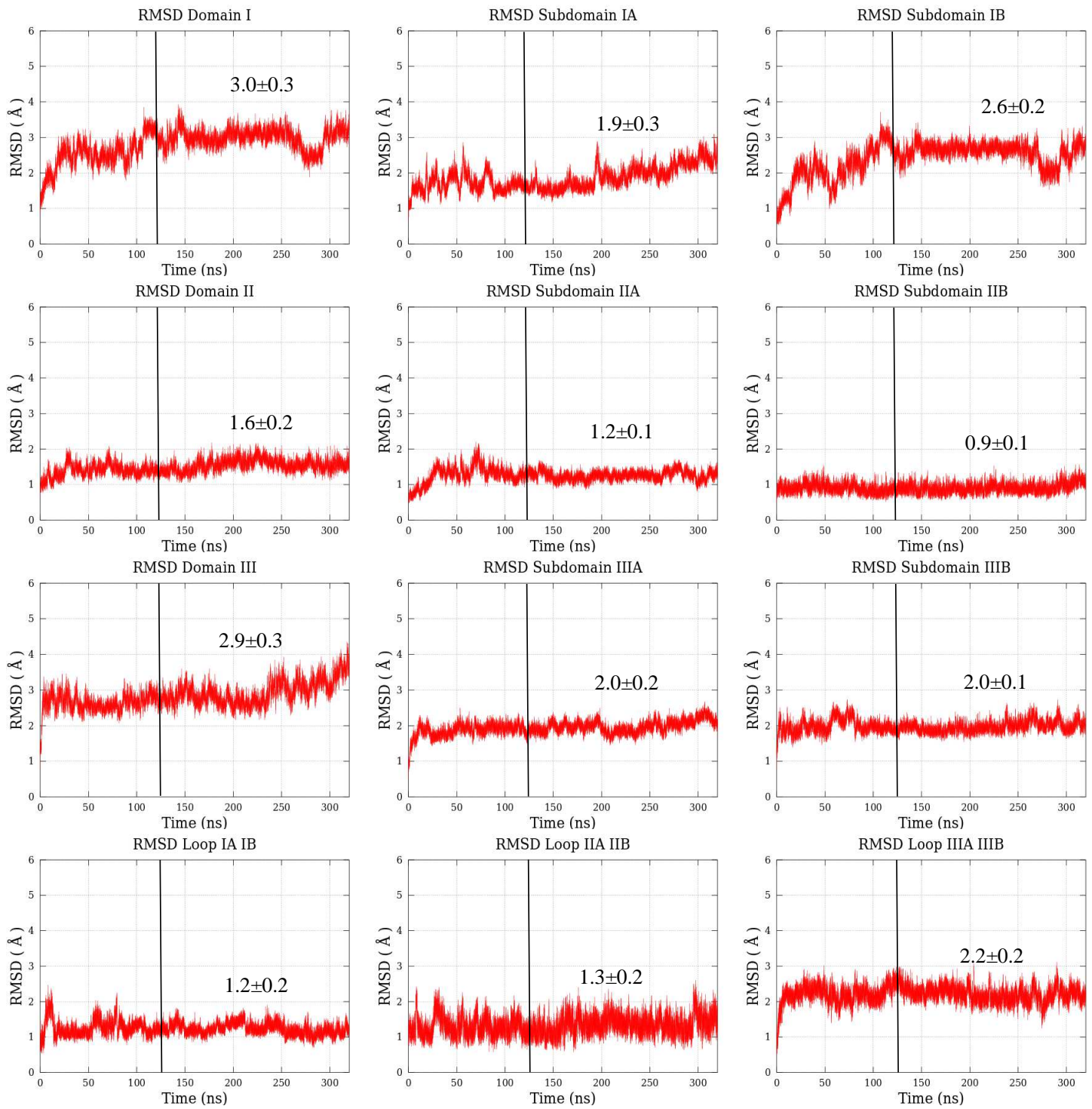
Figure S1 (cont). Time evolution along the STD-b trajectory of the root mean squared deviation (RMSD, in $\AA$ ) computed for the heavy backbone atoms in the three domains, six subdomains, and three subdomain connecting loops with respect to the 3JRY crystal structure (molecule A). Average values and standard deviations for the last $200 \mathrm{~ns}$ are included.
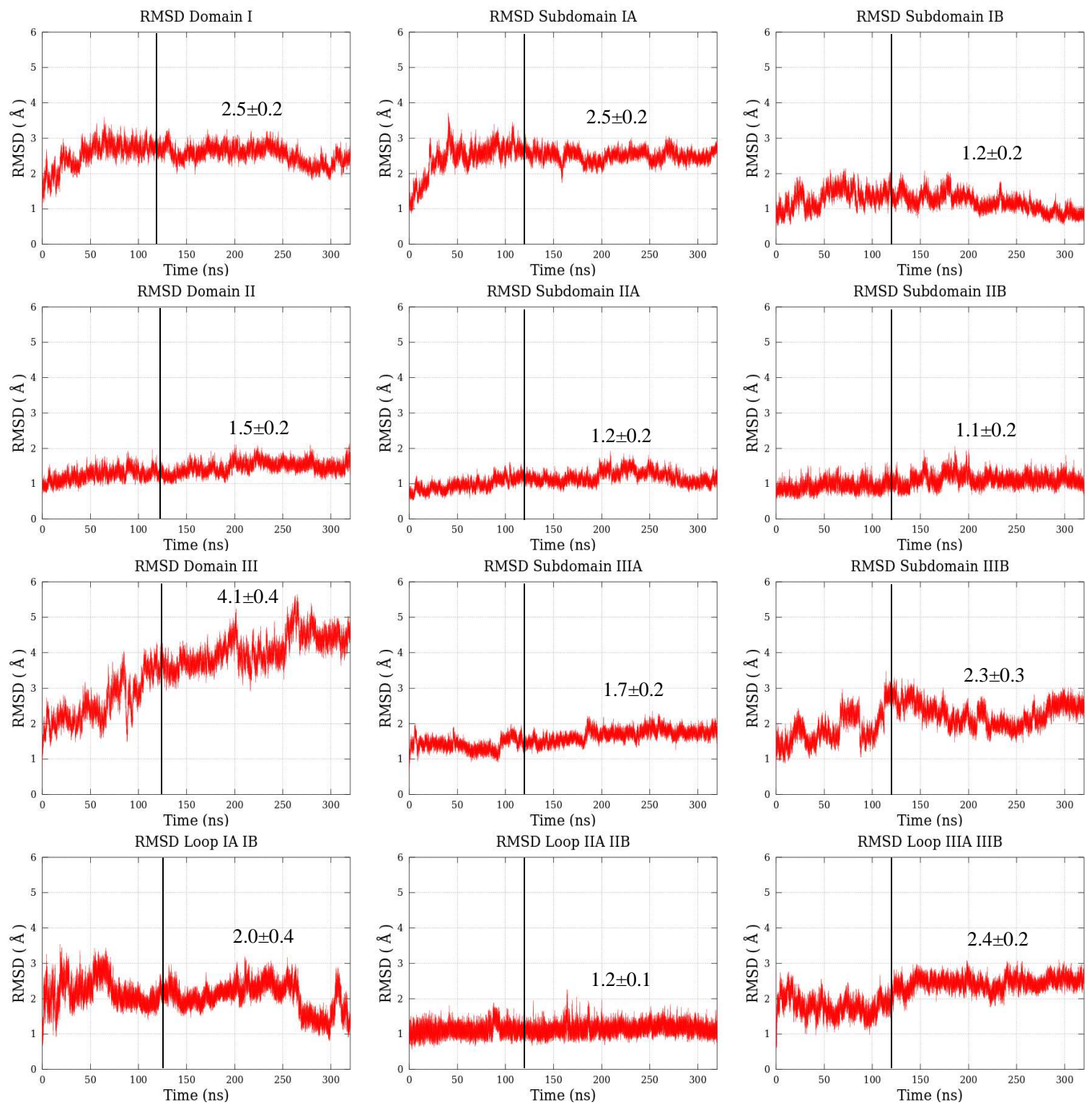
Figure S2. Time evolution along the PK-PH7-a trajectory of the root mean squared deviation (RMSD, in $\AA$ ) computed for the heavy backbone atoms in the three domains, six subdomains, and three subdomain connecting loops with respect to the 3JRY crystal structure (molecule A). Average values and standard deviations for the last $200 \mathrm{~ns}$ are included.
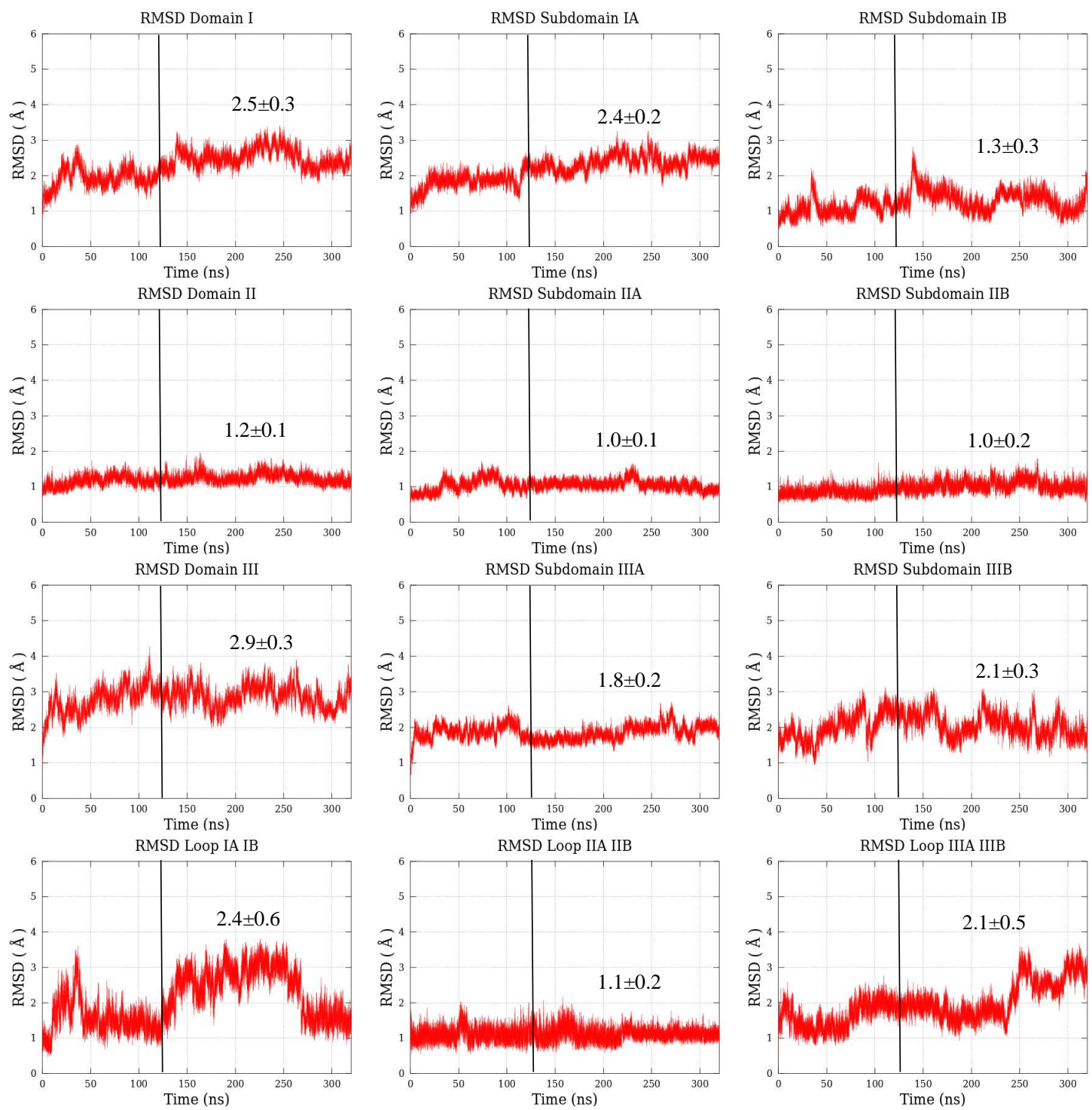
Figure S2 (cont.). Time evolution along the PK-PH7-b trajectory of the root mean squared deviation (RMSD, in $\AA$ ) computed for the heavy backbone atoms in the three domains, six subdomains, and three subdomain connecting loops with respect to the 3JRY crystal structure (molecule A). Average values and standard deviations for the last $200 \mathrm{~ns}$ are included.
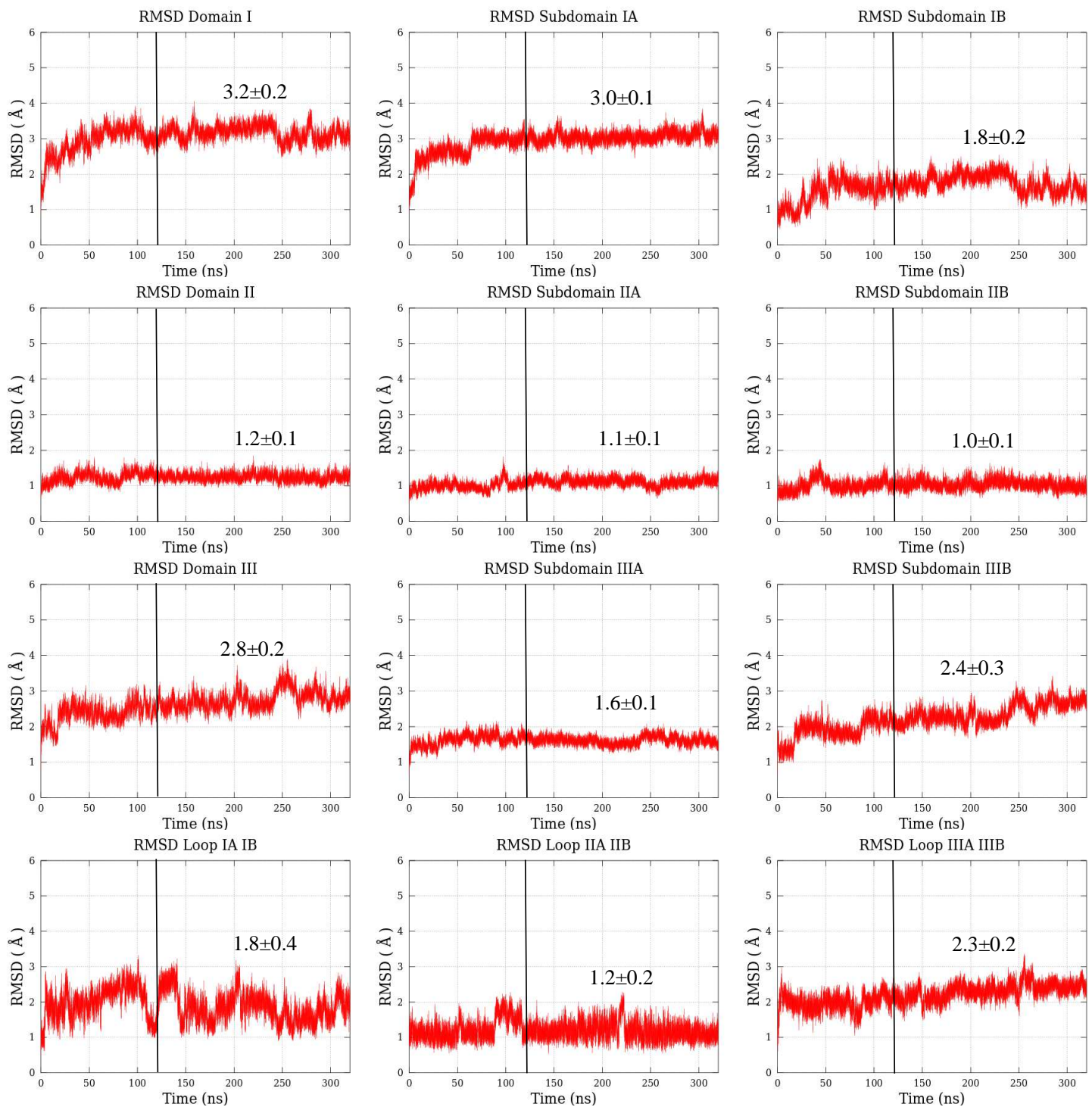
Figure S3. Time evolution along the constant $p H$ trajectory of the root mean squared deviation (RMSD, in $\AA$ ) computed for the heavy backbone atoms in the full protein, three domains, six subdomains, and three subdomain connecting loops with respect to the 3JRY crystal structure (molecule A).
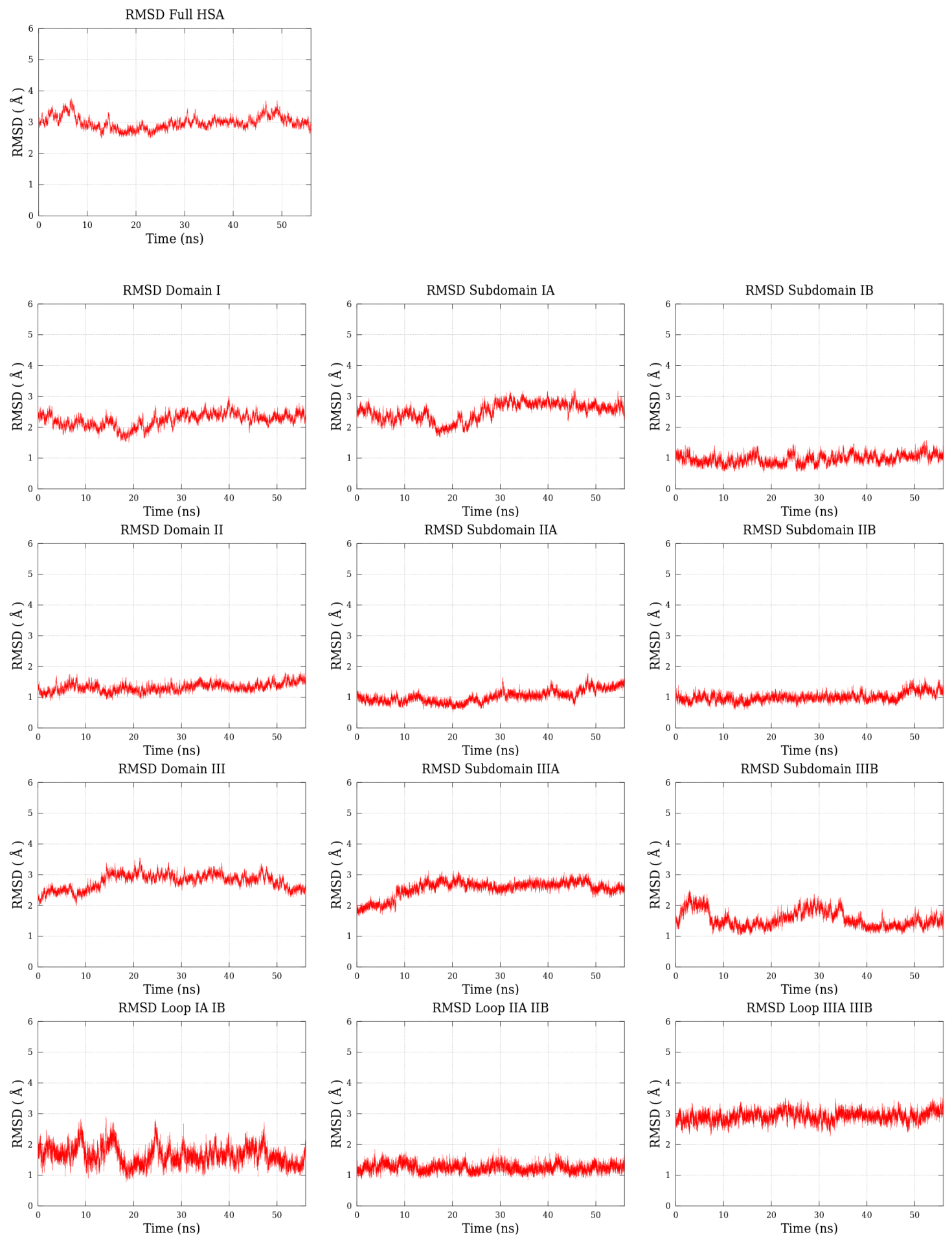
Figure S4. Time evolution along the PK-PH9-a trajectory of the root mean squared deviation (RMSD, in $\AA$ ) computed for the heavy backbone atoms in the three domains, six subdomains, and three subdomain connecting loops with respect to the 3JRY crystal structure (molecule A). Average values and standard deviations for the last $200 \mathrm{~ns}$ are included.
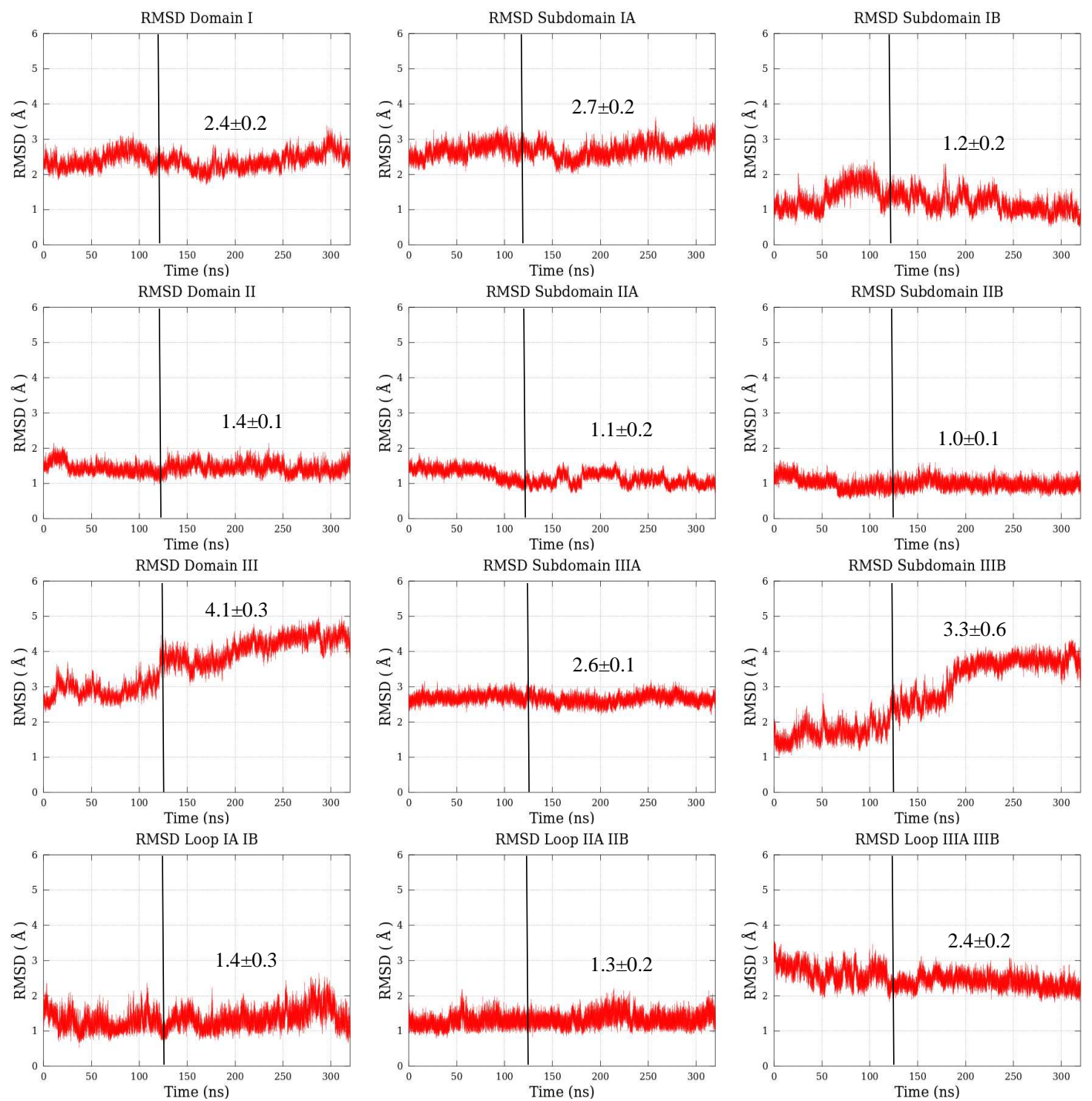
Figure S4 (cont.). Time evolution along the PK-PH9-b trajectory of the root mean squared deviation (RMSD, in $\AA$ ) computed for the heavy backbone atoms in the three domains, six subdomains, and three subdomain connecting loops with respect to the 3JRY crystal structure (molecule A). Average values and standard deviations for the last $200 \mathrm{~ns}$ are included.
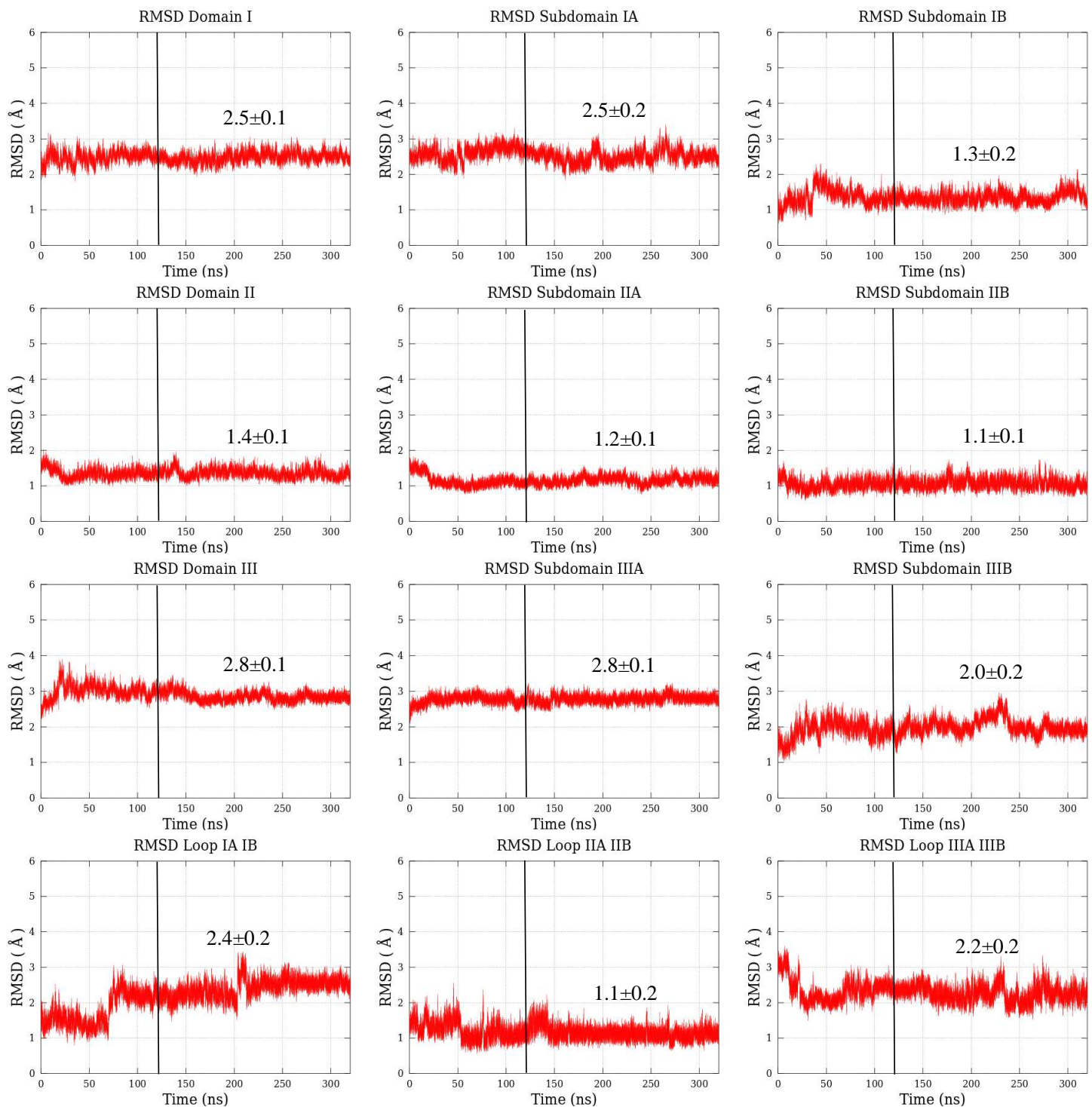
Figure S5. Ribbon model of the last snapshot obtained from the constant-pH simulation (colored defining the six different subdomains) superposed on the ribbon model of the 3JRY crystal structure (in light grey).

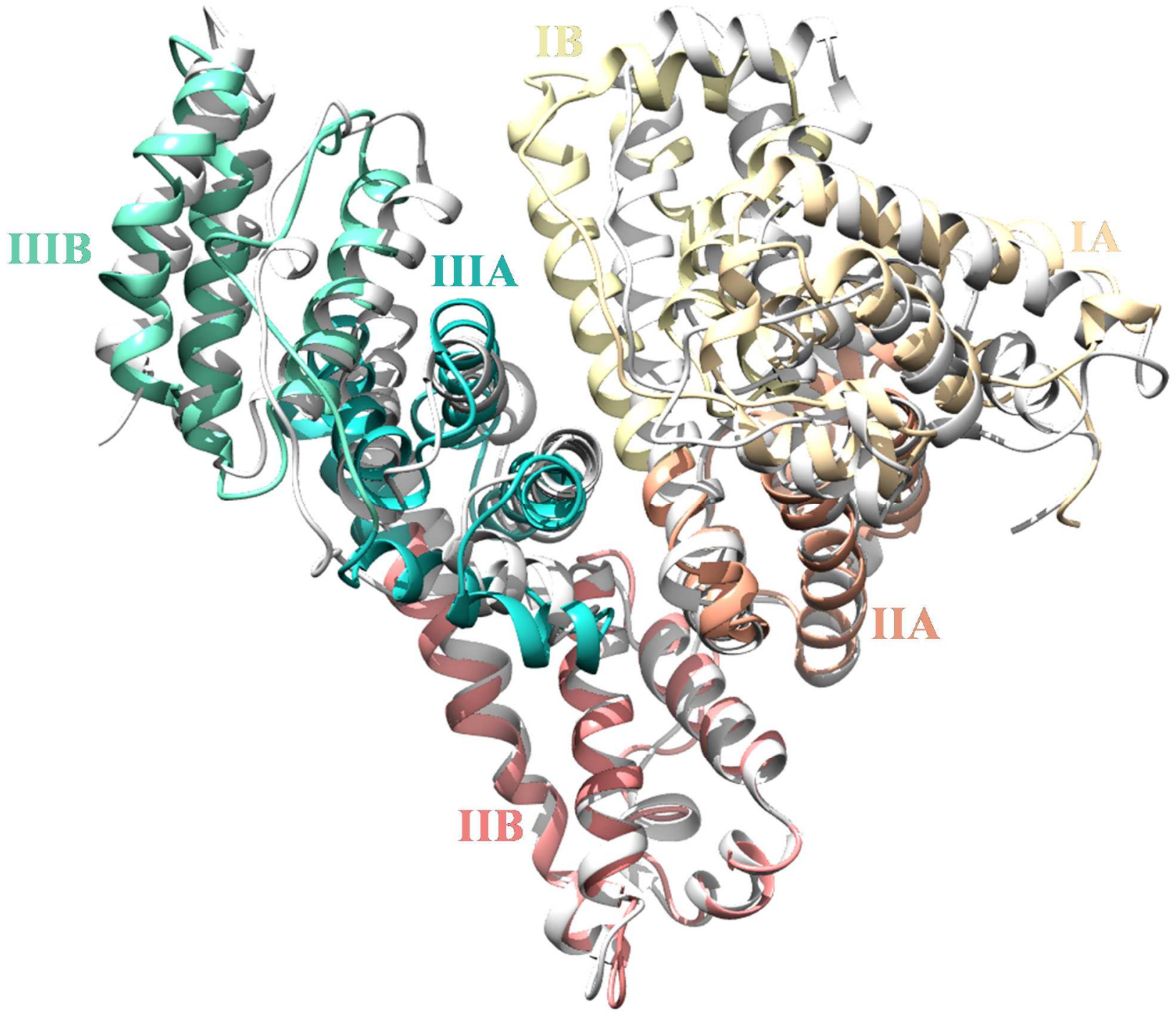

